\title{
The baryon cycle of Seven Dwarfs with superbubble feedback
}

\author{
Mattia Mina ${ }^{1}$, Sijing Shen ${ }^{1}$, Benjamin Walter Keller ${ }^{2}$, Lucio Mayer $^{3}$, Piero Madau ${ }^{4}$, and James Wadsley ${ }^{5}$ \\ ${ }^{1}$ Institute of Theoretical Astrophysics, University of Oslo, 0315 Oslo, Norway \\ e-mail: mattia.mina@astro.uio.no, sijing.shen@astro.uio.no \\ 2 Astronomisches Rechen-Institut, Zentrum für Astronomie der Universität Heidelberg, 69120 Heidelberg, Germany \\ 3 Center for Theoretical Astrophysics and Cosmology, Institute for Computational Science, University of Zurich, \\ Winterthurerstrasse 190, 9057 Zurich, Switzerland \\ ${ }^{4}$ Department of Astronomy and Astrophysics, University of California, 1156 High Street, Santa Cruz, CA 95064, USA \\ 5 Department of Physics and Astronomy, McMaster University, Hamilton, Ontario L8S 4M1, Canada
}

Received 14 September 2020 / Accepted 17 March 2021

\begin{abstract}
We present results from a high-resolution, cosmological, $\Lambda$ CDM simulation of a group of field dwarf galaxies with the "superbubble" model for clustered SN feedback, accounting for thermal conduction and cold gas evaporation. We compared our results to a previous simulation which has the same initial condition and galaxy formation physics (other than SN feedback), but adopts a delayed-cooling model for supernova. The simulated luminous galaxies have blue colors, low star formation efficiencies and metallicities, and high cold gas content, reproducing the observed scaling relations of dwarf galaxies in the Local Volume. Bursty star formation histories and superbubble-driven outflows lead to the formation of kpc-size dark matter (DM) cores when stellar masses reaches $M_{*}>10^{6} M_{\odot}$, similar to previous findings. However, the superbubble model appears more effective in destroying DM cusps than the delayed-cooling model in the previous study, reflecting a higher coupling efficiency of SN energy with the ISM. On larger scale, superbubble-driven outflows have a more moderate impact: galaxies have higher gas content, more extended stellar discs, and a smaller metal-enriched region in the circumgalactic medium (CGM). The two halos with $M_{\text {vir }} \sim 10^{9} M_{\odot}$, which formed ultra-faint dwarf galaxies with the delayed-cooling mode, remain dark due to the different impact of metal-enriched galactic winds from two nearby luminous galaxies, indicating that the formation of faint dwarfs is highly dependent on feedback and environmental effects. The column density distributions of HI, Si II, C IV and O VI as a function of the scaled impact parameter $\left(b / R_{\mathrm{vir}}\right)$ are in good agreement with recent observations of CGM around isolated dwarf galaxies. While $\mathrm{HI}$ is ubiquitous with a covering fraction of unity within the CGM, low and intermediate ions like Si II and C IV are less extended (typically confined within $0.2-0.3 R_{\text {vir }}$ ), and non-detections are common. $\mathrm{O}$ VI is more extended with column density $\mathrm{N}(\mathrm{O} \mathrm{VI}) \gtrsim 10^{13.5} \mathrm{~cm}^{-2}$ within $R_{\text {vir }}$, but its mass is only $11 \%$ of the total CGM oxygen budget, as the diffuse CGM is highly ionised by the UV background. Superbubble feedback produces C IV and O VI column densities that are an order of magnitude higher than those in the previous study using delayed-cooling feedback. Thus, the CGM and DM cores are most sensitive probes of feedback mechanisms.
\end{abstract}

Key words. galaxies: formation - galaxies: dwarf - intergalactic medium - dark matter - methods: numerical

\section{Introduction}

Within the standard $\Lambda$ cold dark matter $(\Lambda \mathrm{CDM})$ model, the Universe today consists of photons and ordinary matter $(\sim 5 \%)$, a cold and collisionless form of matter known as dark matter $(\sim 27 \%)$ and a cosmological constant $\Lambda(\sim 68 \%)$ which describes the dark energy component, responsible for the late-time accelerated expansion. The $\Lambda C D M$ model has been very successful in describing the large-scale properties of the observed Universe. However, small-scales are still a source of unanswered questions.

The first generation of cosmological dark matter-only simulations revealed the existence of severe tensions between theoretical predictions of the $\Lambda \mathrm{CDM}$ model and astronomical observations. In the core-cusp problem, in small and heavily dark matter-dominated galaxies, dark matter halos exhibit central density profiles characterised by an almost constant density core (Oh et al. 2015; Agnello \& Evans 2012; Adams et al. 2014; Amorisco \& Evans 2011; Walker \& Peñarrubia 2011; Battaglia et al. 2008; Flores \& Primack 1994; Moore 1994), which seems to be inconsistent with steeper central densities of dark halos found in dark matter-only simulations of the $\Lambda$ CDM model, such as Dubinski \& Carlberg (1991) and Navarro et al. (1996). Furthermore, several numerical studies, such as Springel et al. (2008), Kuhlen et al. (2009), Stadel et al. (2009), Garrison-Kimmel et al. (2014), and Griffen et al. (2016), have shown how the $\Lambda \mathrm{CDM}$ paradigm predicts the formation of a large number of dark matter subhalos, many more than the actual number of observed satellites orbiting M 31 and the Milky Way, which is the basis of the so-called missing-satellite problem (Moore et al. 1999). In addition, the most massive subhalos found in dark matter-only simulations of Milky Way-mass halos have higher peak rotation velocities than the observed Milky Way satellites, and the fact that these seemingly more massive satellites are not observed in the Local Group is often framed as the 'too-big-to-fail' problem (Boylan-Kolchin et al. 2011, 2012).

While many alternative dark matter models have been proposed to solve the small-scale crisis (see e.g., Bertone et al. 2005; Steffen 2009; Bergström 2009), many recent simulations have shown that environmental effects such as ram pressure and tidal stripping, together with ultraviolet (UV) background heating and stellar feedback, can either regulate gas condensation at the centre of galaxies or eject the gas altogether. Baryonic physics may therefore explain the low star-formation efficiencies 
seen in dwarf galaxies, the low abundance of dwarf galaxies compared to the high number of low-mass DM structures predicted by the $\Lambda \mathrm{CDM}$ model, and provide a solution to the missing satellite problems (Hoeft et al. 2006; Okamoto et al. 2008; Madau et al. 2008; Governato et al. 2010, 2015; Simpson et al. 2013; Munshi et al. 2013; Brooks \& Zolotov 2014; Noh \& McQuinn 2014; Trujillo-Gomez et al. 2015; Sawala et al. 2016; Wetzel et al. 2016). In addition, it was shown that rapid bursts of star formation and outflows due to stellar feedback can dynamically heat the dark matter structure of dwarf galaxies, reducing the central density of dark halos and creating central dark matter cores, providing solutions to the cusp-core problem and related too-big-to-fail problem (Mashchenko et al. 2008; Governato et al. 2010; Pontzen \& Governato 2012; Zolotov et al. 2012; Madau et al. 2014; Chan et al. 2015; Oñorbe et al. 2015; Read et al. 2016; Tollet et al. 2016; Fitts et al. 2017).

These baryonic physical processes have also been shown to be crucial for the reproduction of realistic dwarf galaxies in the Local Volume. Indeed, modern hydrodynamic simulations of dwarf galaxies (e.g., Brooks \& Zolotov 2014; Shen et al. 2014; Oñorbe et al. 2015; Wheeler et al. 2015; Fitts et al. 2017) have achieved great success in reproducing the observed properties, such as stellar mass content (e.g., Behroozi et al. 2013; Moster et al. 2013; Brook et al. 2014; Garrison-Kimmel et al. 2014; Read et al. 2017; Jethwa et al. 2018), cold gas content, star formation history (e.g., Tolstoy et al. 2009; Weisz et al. 2011, 2014; Brown et al. 2014), mass-metallicity relationship (e.g., McConnachie 2012; Kirby et al. 2013, 2014), and stellar, gas kinematics, and morphological properties (e.g., McConnachie 2012) in field and satellite dwarf galaxies in the local Universe. With ever increasing resolution, simulations are now capable of probing the formation of ultra-faint dwarf galaxies (UFDs) and can be used to make predictions for upcoming deep surveys from telescopes such as the Vera C. Rubin Observatory (e.g., Fitts et al. 2017; Revaz \& Jablonka 2018; Wheeler et al. 2019; Wright et al. 2019a; Munshi et al. 2019; Agertz et al. 2020; Applebaum et al., in prep.). All these simulations have confirmed that, at least in the regime of classical dwarfs $\left(M_{\mathrm{vir}} \sim 10^{10} M_{\odot}\right)$, galactic outflows driven by stellar feedback are of critical importance to suppress star formation, reduce the stellar bulge-to-disc ratio, lower the intestellar medium (ISM) metallicity, and enrich the CGM.

Despite the consensus on the importance of galactic winds in dwarf galaxy formation, large uncertainties persist in how the winds are driven. As the ISM physics is typically not resolved in full cosmological simulations (although some simulations are getting close, see e.g., Wheeler et al. 2019; Agertz et al. 2020), subgrid feedback models are often used to alleviate the numerical 'overcooling' problem, whereby energy injected into the ISM is rapidly cooled away because of unresolved ISM structures. Many studies have investigated supernova (SN) feedback, perhaps the most important process to drive galactic wind in dwarf galaxies. Different approaches of subgrid models were explored, ranging from kinetic feedback models where wind mass loading and velocities are predetermined (Springel \& Hernquist 2003; Oppenheimer \& Davé 2006; Dalla Vecchia \& Schaye 2008), to explicit models where radiative cooling is temporarily shut off (Stinson et al. 2006; Agertz et al. 2011; Teyssier et al. 2013), to mechanical feedback where the momentum boost during the unresolved adiabatic phase is calibrated through smallscale ISM simulations and injected into the ISM (Hopkins et al. 2014; Kimm \& Cen 2014; Smith et al. 2018). Simulations of dwarf galaxies in recent years have often adopted one of these approaches for SN feedback. Although many are considered successful in reproducing observations, few attempts have been made to compare these models in full cosmological simulations. It is therefore important to gauge how the evolution of simulated dwarf galaxies (especially at the low mass end) is dependent on feedback models, in order to provide a more robust model with realistic uncertainties for future observations. Moreover, most existing SN feedback models take into account individual SN explosions. However, recent studies such as Nath \& Shchekinov (2013) and Sharma et al. (2014), have pointed out that stars form in clusters and, through multiple repeated SN explosions, generate the so-called superbubbles, which contain hot gas, and the sharp temperature gradients between hot and cold gas make thermal conduction an extremely important process. The evolution of superbubbles and the importance of thermal conduction have been investigated using ISM simulations with direct treatments of thermal conduction (e.g., El-Badry et al. 2019; Steinwandel et al. 2020). However, the resolution requirement for directly modelling conduction is still unattainable for cosmological galaxy formation simulations.

Keller et al. (2014) therefore proposed the superbubble model, where the thermal conduction between cold and hot phases of subresolution multi-phase particles is included, as well as a stochastic evaporation model for cold gas. As shown by Mac Low \& McCray (1988), thermal conduction describes the mass flow from the cold shell into the hot gas, therefore controls how much gas is heated by feedback. Bubble temperature and mass are determined by the evaporation process, along with radiative cooling, without the need for additional free parameters and naturally avoiding the overcooling problem. In this way, superbubbles efficiently convert feedback energy into thermal and kinetic energy in the hot and the cold phases, respectively. Keller et al. $(2015,2016)$ performed the first cosmological simulations of halos with masses of $M_{\mathrm{vir}}>10^{11} M_{\odot}$ with superbubble feedback, and showed that this model can launch strong galactic outflows, effectively regulating star formation from early on $(z>2)$. The simulations with supperbubble-driven outflows reproduce many of the observed properties of $L_{*}$ galaxies. More recently, a new theoretical framework was proposed Keller et al. (2020), where it was shown that outflows from superbubble feedback have higher entropy and are continuously accelerated in the CGM because of to buoyancy. As a consequence, although the winds have higher mass loading and lower velocities, they tend to stay in the CGM over a longer timescale.

In the present work, we extend the investigation to the formation of the low-mass dwarf-galaxy regime for halos with $M_{\text {vir }} \lesssim 10^{10} M_{\odot}$ by re-simulating the group of seven field dwarf galaxies presented in Shen et al. (2014) with the superbubble feedback model from Keller et al. (2014). In the original study, Shen et al. (2014) adopted the blastwave feedback (Stinson et al. 2006) and produced highly realistic dwarf galaxies (two classical dwarfs and two faint dwarfs), including their dark matter cores (Madau et al. 2014). The goal of the present study is to investigate whether the superbubble feedback model, which provides a more accurate description of the underlying physics, can produce realistic isolated dwarf galaxies in the $\Lambda$ CDM framework, without involving an ad hoc delayed cooling prescription. In addition, we compare the superbubble model with the blastwave feedback model, and explore in detail how sensitive the formation and properties of dwarf galaxies are to feedback models.

The paper is organised as follows. In Sect. 2, we present the details of our simulations, briefly summarising the main features of the superbubble model. In Sect. 3, we describe the two 
galaxies that formed within our simulation and we compare their properties to the results of previous numerical studies and to observations. In Sect. 4, we study the properties of the host dark matter halos, with particular focus on the cusp-core transformation induced by stellar feedback. In Sect. 5, we analyse the impact of stellar feedback on the formation of ultra-faint dwarf galaxies. We compare the CGM surrounding the two galaxies to absorption line observations in Sect. 6, and present our conclusions in Sect. 7.

\section{Simulations}

Simulations were performed by using the TreeSPH code GASOLINE2 (Wadsley et al. 2017), with the same initial conditions and numerical setup as in Shen et al. (2014). Briefly, the cosmology we adopt is identified by $\Omega_{\mathrm{b}}=0.042, \Omega_{\mathrm{m}}=0.24$, $\Omega_{\Lambda}=0.76, h=0.73$, and $\sigma_{8}=0.77$. At redshift $z=0$, the simulation box is about $25 \mathrm{Mpc}$ on a side, with an embedded highresolution region of about $2 \mathrm{Mpc}$ on a side. The latter region contains 6 million dark matter particles and an equal number of smoothed particle hydrodynamics (SPH) particles, with mass resolution of $m_{\mathrm{dm}}=1.6 \times 10^{4} M_{\odot}$ and $m_{\mathrm{sph}}=3.3 \times 10^{3} M_{\odot}$, respectively. The gravitational softening length, $\epsilon_{\mathrm{G}}$, is set to $86 \mathrm{pc}$ (physical) from $z=9$ to present, while it evolves as $1 /(1+z)$ from the initial redshift of $z=129$ to $z=9$. The smoothing length of SPH particles is allowed to shrink up to $0.1 \epsilon_{\mathrm{G}}$. The simulation includes radiation from a UV background from Haardt \& Madau (2012) and cooling from primordial and metal species over a temperature range of $T=10-10^{9} \mathrm{~K}$ as described in Shen et al. (2010). 1959):

The star formation recipe follows the Schmidt law (Schmidt

$\frac{\mathrm{d} \rho_{*}}{\mathrm{~d} t}=0.1 \frac{\rho_{\mathrm{gas}}}{t_{\mathrm{dyn}}} \propto \rho^{1.5}$,

where $\rho_{*}$ and $\rho_{\text {gas }}$ are respectively the stellar and gas densities, while $t_{\text {dyn }}$ represents the local dynamical time. Star formation occurs stochastically when gas simultaneously reaches temperatures $T<10^{4} \mathrm{~K}$ and exceeds a given density threshold of $n_{\mathrm{H}}=100$ atoms $\mathrm{cm}^{-3}$. A single star particle represents a simple stellar population with its own age, metallicity and initial mass function (IMF). Initially, the mass of each star particle is $m_{*}=10^{3} M_{\odot}$ and its IMF is determined according to Kroupa (2001). As each stellar population evolves, energy, mass and metals are injected into the ISM through Type Ia and Type II SNe and stellar winds. While the stellar wind and Type Ia SN feedback models remain the same as in Shen et al. (2014) as described in Stinson et al. (2006) (without cooling shut off), Type II SN feedback follows the superbubble model described in Keller et al. (2014). Briefly, the novelty of this model lies in its three main components: thermal conduction, stochastic evaporation of cold gas, and subresolution multi-phase particles.

Thermal conduction is an important process in the accurate modelling of the evolution of superbubbles. In the presence of temperature gradients, the heat flux $Q$ is computed as $Q=-\kappa \nabla T$, where $T$ is the temperature, and $\kappa(T)$ is the conduction coefficient. $\kappa(T)$ can be approximated by $\kappa_{0} T^{5 / 2}$ in which $\kappa_{0}=6.1 \times 10^{-7} \mathrm{erg} \mathrm{s}^{-1} K^{-7 / 2} \mathrm{~cm}^{-1}$ in cases where magnetic fields are not present (Cowie \& McKee 1977). As heat transfer is mediated by electrons, a mass flux from cold to hot phase (i.e. evaporation of cold gas) must also be present in order to not generate currents in the fluid. However, this process takes place on spatial scales that are too small to be resolved. Therefore,
Keller et al. (2014) introduced a subgrid treatment for evaporation of cold gas. Given the internal temperature $T$ and the surface area $A$ of the hot bubble, the mass variation is computed according to the following formula:

$\frac{\mathrm{d} M_{\mathrm{b}}}{\mathrm{d} t}=\frac{4 \pi \mu}{25 k_{\mathrm{B}}} \kappa_{0} \frac{\Delta T^{5 / 2}}{\Delta x} A$,

where $\Delta x$ corresponds to the thickness of the boundary layer between cold and hot gas. In practise, we adopt an area estimate of $A=\left(6 \pi h^{2}\right) / N_{\text {hot }}$ and $\Delta x=h$, where $h$ is the smoothing length of a given hot particle in the bubble and $N_{\text {hot }}$ is the number of neighbouring hot particles. Cold gas particles are evaporated into the hot phase stochastically by converting Eq. (2) into a probability per time-step. Evaporated gas particles then receive half of the thermal energy of the hot gas particle that causes their evaporation.

At early stages of the formation of a bubble, or in lowresolution simulations, resolution limits often prevent a proper estimation of temperature and density, leading once again to an overcooling problem. In the superbubble model, rather than shutting down cooling for a given amount of time, as done by Stinson et al. (2006), fluid elements are allowed to temporarily enter a multi-phase state. Numerically speaking, particles are defined as cold if their temperature falls below a given threshold of $T_{\mathrm{th}}=10^{5} \mathrm{~K}$. During feedback events, energy is dumped into surrounding gas and particles enter a two-phase state, carrying two values of mass and energy for the hot and the cold phases, which are assumed to be in pressure equilibrium. Thus, each particle has total mass and energy:

$m=m_{\text {hot }}+m_{\text {cold }}$,

$E=u_{\text {hot }} m_{\text {hot }}+u_{\text {cold }} m_{\text {cold }}$,

where $u$ corresponds to the internal energy per unit of mass. By means of Eqs. (3) and (4), the density of the phase of each particle is then computed by assuming pressure equilibrium. $P d V$ work onto multi-phase particles are distributed to the two phases according to their current energy fractions, thus preserving pressure equilibrium. At each time-step, the mass flux between the two phases is determined by the evaporation rate given by Eq. (2). More explicitly,

$\frac{\mathrm{d} M_{\mathrm{b}}}{\mathrm{d} t}=\frac{16 \pi \mu}{25 k_{\mathrm{B}}} \kappa_{0} T_{\mathrm{hot}}^{5 / 2} h$,

where $h$ is the SPH smoothing length. Once the temperature of the hot phase falls below $T_{\mathrm{th}}$, or the cold phase evaporates entirely, the given particle returns to a single-phase state.

Metal enrichment is modelled in the same way as in Shen et al. (2014). Briefly, the yields of oxygen and iron are tracked separately according to Raiteri et al. (1996), and converted to alpha-elements and iron-peak elements, respectively, assuming solar abundances patterns (Asplund et al. 2009). Turbulent mixing of metals (and thermal energy) is modelled with a variant of the Smagorinsky (1963) turbulent diffusion model, as described in detail in Shen et al. (2010).

\section{The simulated dwarfs}

At the present day, two field dwarf galaxies have formed in our simulation, as can be seen in Fig. 1, which shows the integrated dark matter and gas density field. Following the same naming convention as Shen et al. (2014), the two dwarfs Bashful and Doc inhabit the two most massive dark matter halos found in 



Fig. 1. Projected dark matter (left panel) and gas (right panel) density fields of the simulated dwarfs at $z=0$, in a square region of $500 \mathrm{kpc}$ on a side within the zoomed-in region. The projections are centred on Bashful, the most massive among the two simulated dwarfs. The virial radii of the Bashful and Doc are marked by the white circles.

Table 1. Properties of the simulated dwarf galaxies at the present day.

\begin{tabular}{lccccccccccc}
\hline \hline Name & $\begin{array}{c}M_{\text {vir }} \\
\left(M_{\odot}\right)\end{array}$ & $\begin{array}{c}R_{\text {vir }} \\
(\mathrm{kpc})\end{array}$ & $\begin{array}{c}R_{\mathrm{c}} \\
(\mathrm{kpc})\end{array}$ & $\begin{array}{c}V_{\max } \\
\left(\mathrm{km} \mathrm{s}^{-1}\right)\end{array}$ & $\begin{array}{c}M_{*} \\
\left(M_{\odot}\right)\end{array}$ & $\begin{array}{c}M_{\mathrm{gas}} \\
\left(M_{\odot}\right)\end{array}$ & $\begin{array}{c}M_{\mathrm{HI}} \\
\left(M_{\odot}\right)\end{array}$ & $f_{\mathrm{b}}$ & $\langle[\mathrm{Fe} / \mathrm{H}]\rangle$ & $M_{V}$ & $B-V$ \\
\hline Bashful & $3.57 \times 10^{10}$ & 85.25 & $3.45_{-0.17}^{+0.16}$ & 49.30 & $1.43 \times 10^{8}$ & $1.27 \times 10^{9}$ & $7.03 \times 10^{7}$ & 0.039 & -1.22 & -15.49 & 0.66 \\
Doc & $1.45 \times 10^{10}$ & 63.23 & $1.86_{-0.07}^{+0.07}$ & 39.40 & $1.48 \times 10^{7}$ & $3.66 \times 10^{8}$ & $2.72 \times 10^{7}$ & 0.026 & -1.55 & -13.58 & 0.35 \\
Dopey & $3.27 \times 10^{9}$ & 38.44 & - & 22.37 & - & $4.39 \times 10^{7}$ & $4.66 \times 10^{3}$ & 0.013 & - & - & - \\
Grumpy & $1.71 \times 10^{9}$ & 30.98 & - & 21.45 & - & $9.28 \times 10^{6}$ & $4.63 \times 10^{3}$ & 0.005 & - & - & - \\
\hline
\end{tabular}

Notes. The different columns indicate, respectively, the name of the galaxy, virial mass, virial radius, dark matter core radius, maximum circular velocity, stellar mass, gas mass, H I mass, baryon fraction $f_{\mathrm{b}} \equiv\left(M_{*}+M_{\mathrm{gas}}\right) / M_{\mathrm{vir}}$, mean stellar metallicity, $V$-band magnitude, and $B-V$ colour. We note that we define the virial radius as the radius enclosing a mean density of 93 times the critical density.

the high-resolution region. Other dark halos present in the field, have also accreted gas during the evolution of the simulated Universe. However, in their case, gas was not able to cool enough to ignite star formation. In particular, smaller dwarf galaxies found in Shen et al. (2014), such as Dopey and Grumpy, are not able to form here. This suggests that the formation of ultra-faint dwarf galaxies is highly sensitive to external feedback mechanisms, which we discuss in Sect. 5. Some global properties of the four most massive halos in the simulation at $z=0$ are listed in Table 1. All of them are field dwarf galaxies with the nearest massive halo of $M_{\mathrm{vir}}=2.5 \times 10^{12} M_{\odot}$ more than $3 \mathrm{Mpc}$ away.

\subsection{Stellar mass-halo mass relationship}

At redshift $z=0$, Bashful and Doc have stellar masses of $M_{*}=1.4 \times 10^{8} M_{\odot}$ and $M_{*}=1.5 \times 10^{7} M_{\odot}$, corresponding to stellar mass fractions $M_{*} / M_{\text {vir }}$ of 0.004 and 0.001 , respectively. Figure 2 shows the stellar mass-halo mass relation (SMHM) for the two dwarfs, together with abundance matching results from Behroozi et al. (2013) and Moster et al. (2013), and more recent relations derived from low-mass Local Group dwarf galaxies (Brook et al. 2014; Garrison-Kimmel et al. 2014), and results from Read et al. (2017) where the stellar masses of isolated dwarf galaxies are derived from fitting their rotation curves. As shown in Fig. 2, the stellar mass fractions of Bashful and Doc are in excellent agreement with abundance matching results from Moster et al. (2013) and Brook et al. (2014). Both Bashful and Doc appears slightly above the
SMHM of Garrison-Kimmel et al. (2014), but Doc lies below the Behroozi et al. (2013) relation and the data points form Read et al. (2017). Given the large uncertainties associated with the SMHM relation at low-mass regimes, our results are in general very consistent with abundance matching results. Comparison with the results from the simulations with the blastwave feedback in Shen et al. (2014) shows that Bashful has a similar stellar mass, whereas the stellar mass of Doc has reduced by more than a factor of two, indicating that the superbubble feedback is more effective at regulating star formation for low-mass galaxies.

We used the Padova simple stellar populations models (Marigo et al. 2008; Girardi et al. 2010) to compute broadband luminosities. Bashful and Doc have $V$-band magnitudes of $M_{V}=$ -15.49 and -13.58 , respectively. If they were to be observed today, with values of $B-V$ of 0.66 for Bashful and 0.35 for Doc, they would appear blue and they would be classified as dwarf irregulars (dIrrs). At $z=0$, in our simulation volume, the two dwarfs are located within $160 \mathrm{kpc}$ from each other.

\subsection{Star formation history}

In the left panel of Fig. 3, we plot the star formation histories (SFHs) of the two simulated dwarfs. Both Bashful and Doc present an extended SFH characterised by stochastic bursts, each followed by a period of quiescence. Although photo-heating from reionisation suppresses gas accretion, for halos with masses of Bashful and Doc, the primordial gas is able to cool down 




Fig. 2. Present-day stellar mass fraction of dwarf galaxies. The filled coloured circles represent Bashful (red) and Doc (blue) simulated with the supebubble feedback. The empty circles with coloured edges correspond to Bashful (red), Doc (blue), Dopey (purple) and Grumpy (green) simulated in Shen et al. (2014), with the blastwave feedback model. The empty grey squares represent the sample of isolated dwarf galaxies analysed in Read et al. (2017). The black lines report the low-mass extrapolations of the SMHM relation provided by Behroozi et al. (2013) (solid), Moster et al. (2013) (dashed), Brook et al. (2014) (dot-dashed), and Brook et al. (2014) (dotted).

its temperature via Compton and radiative cooling and condense at the centre of the gravitational potential well. As gas cools further, stars are ignited and, along with the first bursts, $\mathrm{SN}$-driven outflows deplete the central regions of star-forming material. A short quiescence phase follows, during which cold gas is accreted towards the centre and a new cycle starts again. Bursty star formation is commonly seen in simulations with explicit, strong SN feedback models (e.g., Governato et al. 2012; Teyssier et al. 2013; Shen et al. 2014; Oñorbe et al. 2015; Fitts et al. 2017; Wright et al. 2019a), which often leads to rapid change of inner gravitational potential wells and to a flattening of central dark matter profiles (e.g., Governato et al. 2010; Pontzen \& Governato 2012; Madau et al. 2014). Dark matter core formation is also seen in our simulation, and we discuss this in more detail in Sect. 4.

While the SFH of Bashful shows no sign of long interruption periods, Doc shows signs of strong suppression of star formation over a longer period of time, and completely stops forming stars for a $\gtrsim 3$ Gyr period between $0.5<z<0.1$, after which, it starts forming stars again. Investigations of resolved stellar populations in the nearest dwarf galaxies found that bursts of star formation can be separated in time by tens or hundreds of millions of years to a few gigayears (e.g., Tolstoy et al. 2009). This result is similar to that of Wright et al. (2019a), who simulated dwarf galaxies in the mass range of $M_{\mathrm{vir}} \sim 10^{9-10} M_{\odot}$ and found that a large fraction of them $(\sim 20 \%)$ show at least one long period of quiescence. In their work, the dwarfs resuming start formation after a long period of quiescence tend to have a higher-than-average ratio of $M_{\mathrm{HI}} / M_{*}$, and start forming stars again after experiencing interaction with streams or filaments of gas from the cosmic web or nearby mergers. This appears to also be the case in Doc, because the whole system is merging towards $z=0$.

In the right panel of Fig. 3, we plot the cumulative star formation histories of the two dwarfs. For comparison, we plot in the same figure cumulative star formation histories of individual dwarf irregular galaxies (dIrrs) found in the ACS Nearby Galaxy Survey Treasury (ANGST) sample, previously analysed in Weisz et al. (2011), together with those of the dwarfs found in Shen et al. (2014). Both Bashful and Doc are actively forming stars in the present epoch of the simulation, which is consistent with observations of field dwarf galaxies. For instance, $80 \%$ of the galaxies in the ANGST catalogue are actively forming stars, and Geha et al. (2012) found that field dwarfs with a stellar mass in the range $10^{7} M_{\odot}<M_{*}<10^{9} M_{\odot}$ that are not forming stars today are extremely rare. Weisz et al. (2011) found that, on average, $50 \%$ of the stellar mass of a dwarf galaxy is formed by redshift $z \sim 2$, while $60 \%$ is formed prior to redshift $z \sim 1$. As in the case of Shen et al. (2014), both Bashful and Doc have SFHs that are highly compatible with those in the ANGST catalogue.

In our work, Doc exhibits a similar trend to the corresponding dwarf in Shen et al. (2014) in terms of cumulative star formation history, even though it exhibits fewer and generally smaller star formation peaks, and longer periods of quiescence compared to the case of the blastwave model. However, early star formation in Bashful appears to be more suppressed by the superbubble feedback, such that it forms half of its stellar mass by $z \lesssim 1$, about 3 Gyr later than Bashful in Shen et al. (2014). This result, together with the fact that Doc has less stellar mass and longer quiescent periods, indicates that the superbubble feedback is more effective for low-mass galaxies with $M_{*} \leq 10^{7} M_{\odot}$, although we note that the SFR in low-mass galaxies like these may be subject to strong stochastic effects (Keller et al. 2019).

\subsection{Stellar distribution and kinematics}

Both Bashful and Doc exhibit exponential stellar discs at the present day. Solid lines in Fig. 4 (left panel) correspond to 1D line-of-sight stellar surface densities as a function of the radial distance from the centre for Bashful and Doc. The dashed lines correspond to exponential fits $\left(I(R>1 \mathrm{kpc})=I_{\mathrm{d}} \exp \left(-R / R_{\mathrm{d}}\right)\right)$ to their stellar discs, at radii in the range of $1<R<5 \mathrm{kpc}$. The scale lengths of the two stellar discs are $R_{\mathrm{d}}=(2.07 \pm 0.036) \mathrm{kpc}$ and $R_{\mathrm{d}}=(0.88 \pm 0.023) \mathrm{kpc}$, for Bashful and Doc, respectively. As in Shen et al. (2014), the two dwarfs show no sign of a central bulge and the stellar discs extend to $5 \mathrm{kpc}$ from the centre. There is a signature of a stellar core also in the stellar density profile for Doc similar to that in Shen et al. (2014), which is also seen in the observed dIrr galaxy WLM (Leaman et al. 2012). It is worth noting that the scale length of Bashful is more than twice that found by Shen et al. (2014) with the blastwave feedback $\left(R_{\mathrm{d}}=0.97 \mathrm{kpc}\right)$. One possible explanation is that star formation in Bashful is shifted to later times by the superbubble feedback. The ISM and stellar populations are generally more compact in high-redshift galaxies because of more frequent merger events, and the suppression of high- $z$ star formation activities leads to larger discs (Brook et al. 2012). Moreover, similar to dark matter particles, stars are also collisionless particles. Thus, the same mechanism that causes central dark matter particles to migrate outwards (and thus creates dark matter cores) is also responsible for dynamical heating of the stellar disc. As we show in Sect. 4, superbubble feedback also leads to rapid fluctuations of the central potential well, resulting in a twice-as-large DM core in Bashful. The strong correlation between DM core size and stellar-disc scale length is consistent with previous suggestions by Pontzen \& Governato (2012), and also with observations (Gentile et al. 2009).

In the right panel of Fig. 4, we instead plot the stellar halflight radius, denoted by $R_{1 / 2}$, versus the stellar mass of the simulated dwarfs, at $z=0$. Bashful and Doc have $R_{1 / 2}$ of 

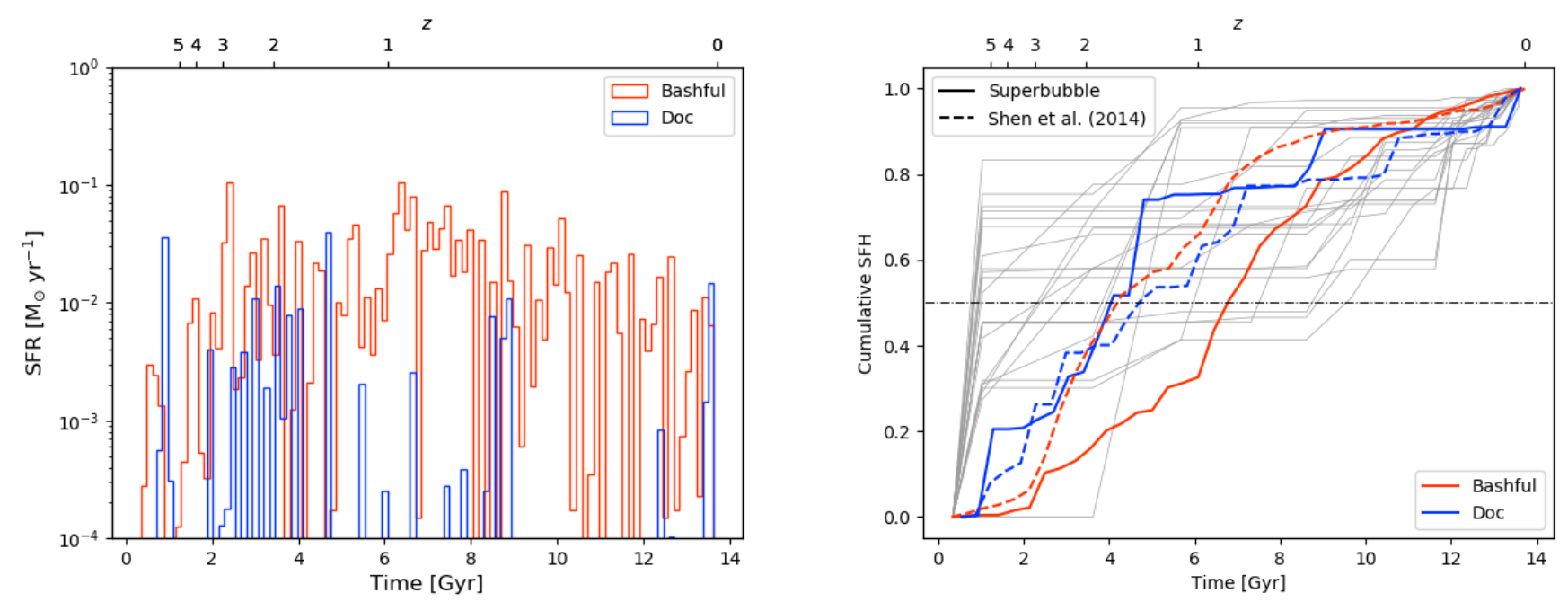

Fig. 3. Left panel: star formation history of Bashful (red) and Doc (blue), averaged a over time intervals of 140 Myr. Right panel: cumulative star formation history of the simulated dwarf galaxies, with total stellar mass normalised to unity. The solid coloured lines represent Bashful (red) and Doc (blue) in our simulation, while the dashed coloured lines correspond, with the same colours, to the dwarfs galaxies simulated in Shen et al. (2014). The grey solid lines are the cumulative star formation histories of individual dwarf irregulars in the ANGST sample (Weisz et al. 2011).
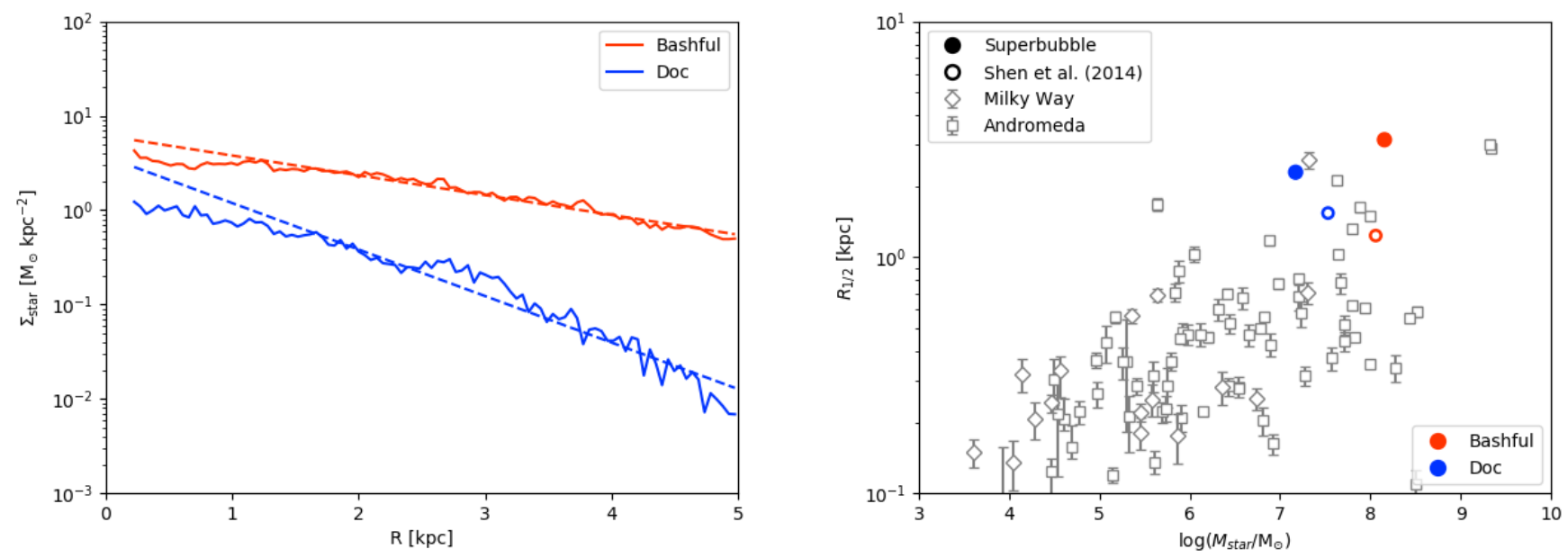

Fig. 4. Left panel: 1D line-of-sight stellar surface density profiles of Bashful (red) and Doc (blue), represented by the solid lines. The dashed lines show the exponential fit of their stellar discs, $I(R>1 \mathrm{kpc})=I_{\mathrm{d}} \exp \left(-R / R_{\mathrm{d}}\right)$, with a characteristic scale of $R_{\mathrm{d}}=(2.07 \pm 0.036) \mathrm{kpc}$ for Bashful and $R_{\mathrm{d}}=(0.88 \pm 0.023) \mathrm{kpc}$ for Doc. Right panel: stellar half-light radius-mass relation of the simulated dwarfs at $z=0$. The filled coloured circles represent Bashful (red) and Doc (blue) in our simulation. The empty circles with coloured edges correspond to Bashful (red) and Doc (blue) simulated in Shen et al. (2014), with the blastwave feedback model. The other empty symbols represent satellite galaxies of the Milky Way (diamonds) and Andromeda (squares), from the original sample of Local Group galaxies published by McConnachie (2012).

3.15 and $2.29 \mathrm{kpc}$, respectively. Both are consistent with (but slightly higher than) those of satellites of the Milky Way and Andromeda galaxies (McConnachie 2012). Similar to the disc scale lengths, $R_{1 / 2}$ from superbubble feedback appears to be significantly larger than those found by Shen et al. (2014) with the delayed cooling model, especially in the case of Bashful. This is again due to the late star formation history and stronger dynamical heating from the superbubble feedback, as discussed above.

In Fig. 5, we show the $V$-band luminosity of the simulated dwarfs against their 1D line-of-sight (LOS) stellar velocity dispersion $\left(\sigma_{*}\right)$, together with the observational data of the dwarf galaxies within the Local Group compiled in McConnachie (2012). The velocity dispersion for each galaxy was computed along three orthogonal lines of sight for all stars within 2 disc scale lengths $\left(R_{\mathrm{d}}\right)$. The $\sigma_{*}$ of Bashful and Doc are $20.8 \mathrm{~km} \mathrm{~s}^{-1}$ and $14.8 \mathrm{~km} \mathrm{~s}^{-1}$, respectively, and the $L_{V}-\sigma_{*}$ relations of Bashful and Doc appear highly consistent with observations. There is no systematic difference in stellar kinematics between the superbubble and blastwave models. As in Shen et al. (2014), we also found that $\sigma_{*}$ increases with stellar age. Stars are born kinematically cold and heated by galaxy mergers and outflows. As larger galaxies often form earlier, have higher star formation rates, and undergo more merger events, it is reasonable to expect that they have larger velocity dispersions. It has also been shown that for satellite galaxies, tidal stripping can decrease $\sigma_{*}$ significantly when dIrrs accrete onto larger galaxies and are transformed into dwarf spheroidal galaxies (dSphs) (e.g., Łokas et al. 2011; Brooks \& Zolotov 2014), but this process is less relevant for field dwarf galaxies such as those investigated here. We also measured the stellar rotational velocities $\left(v_{\text {rot, } *}\right)$ by taking the azimuthal velocities at $2 R_{\mathrm{d}}$, and found that $v_{\mathrm{rot}, *}$ is $11.1 \mathrm{~km} \mathrm{~s}^{-1}$ and $13.6 \mathrm{~km} \mathrm{~s}^{-1}$ for Bashful and Doc, respectively. Thus, the ratio between rotational velocity and velocity dispersion, $\left(v_{\mathrm{rot}} / \sigma\right)_{*}$, is 0.53 and 0.92 for Bashful and Doc, 


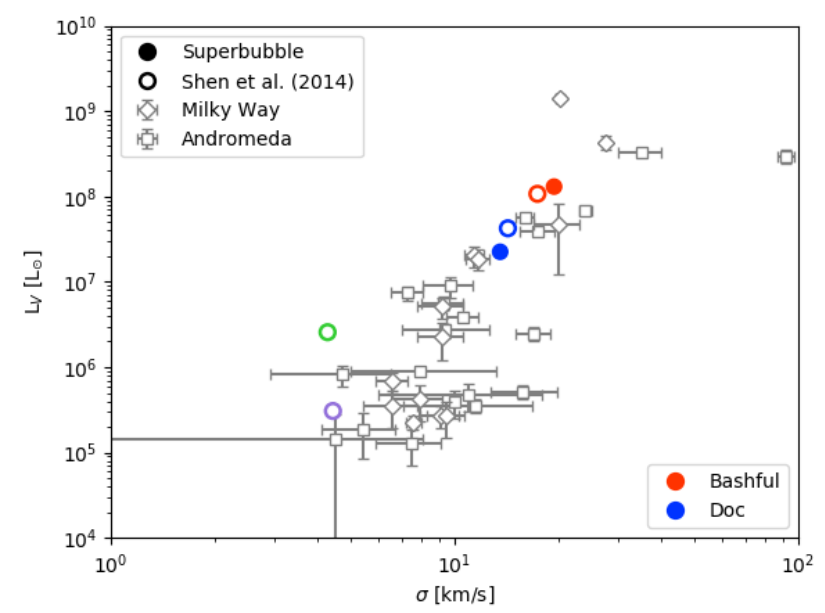

Fig. 5. $V$-band luminosity versus stellar 1D line-of-sight velocity dispersion of the two simulated dwarfs. The $V$-band luminosity was computed using the Padova simple stellar populations models (Marigo et al 2008; Girardi et al. 2010). The filled coloured circles represent Bashful (red) and Doc (blue) in our simulation. The empty circles with coloured edges correspond to Bashful (red), Doc (blue), Dopey (purple) and Grumpy (green) simulated in Shen et al. (2014), with the blastwave feedback model. The empty symbols show the observed $L_{V}-\sigma$ relation for satellite galaxies of the Milky Way (diamonds) and Andromeda (squares), from the original sample of Local Group galaxies published by McConnachie (2012).

respectively, which indicates that the stellar discs are pressuredominated $\left(\left(v_{\text {rot }} / \sigma\right)_{*} \lesssim 1\right)$. This is also consistent with observations of faint dIrrs $\left(v_{\text {rot }} \lesssim 20 \mathrm{~km} \mathrm{~s}^{-1}\right)$ in the Local Volume, where a large fraction of them are found to be dispersion-dominated.

\subsection{Cold gas properties}

As listed in Table 1, at $z=0$, Bashful and Doc have total mass in neutral hydrogen $M_{\mathrm{HI}}=7.03 \times 10^{7} M_{\odot}$ and $2.72 \times 10^{7} M_{\odot}$, respectively. Both values are approximately twice larger than those found for the corresponding dwarfs by Shen et al. (2014) with the blastwave SN feedback model, although the stellar masses are comparable or smaller. It appears that the superbubble feedback is able to retain more cold gas within the galaxies while regulating star formation, and this can be seen in Fig. 6, where we plot the relation between $\mathrm{H}$ I gas mass and $V$-band luminosity for the two simulated dwarfs, together with the data sample published in McConnachie (2012). Bashful and Doc appear to be consistent with the observed dwarfs in the Local Group. The total amount of cold gas increases with stellar luminosity, because more massive galaxies can sustain galactic fountains and re-accrete gas previously blown away by SN feedback; cold gas is also less susceptible to photoionisation heating from the UV background. Nevertheless, we find that the ratio of H I gas mass over stellar mass generally decreases with increasing stellar mass, that is, smaller galaxies are more gas rich. For example, the ALFALFA extragalactic H I survey found more than half of the galaxies of their sample have a H I gas mass-to-stellar mass ratio $M_{\mathrm{HI}} / M_{*}>1$, in particular galaxies with $M_{*} \lesssim 10^{7} M_{\odot}$ Huang et al. (2012). The $M_{\mathrm{HI}} / M_{*}$ of Bashful and Doc are 0.49 and 1.83 for Bashful and Doc, respectively, which is in excellent agreement with the ALFALFA observations.

In contrast to stellar kinematics, the cold gas discs of these systems appear to be rotationally supported. At $z=0$, Bashful and Doc have HI velocity dispersion $\left(\sigma_{\mathrm{HI}}\right)$ of $11.8 \mathrm{~km} \mathrm{~s}^{-1}$ and

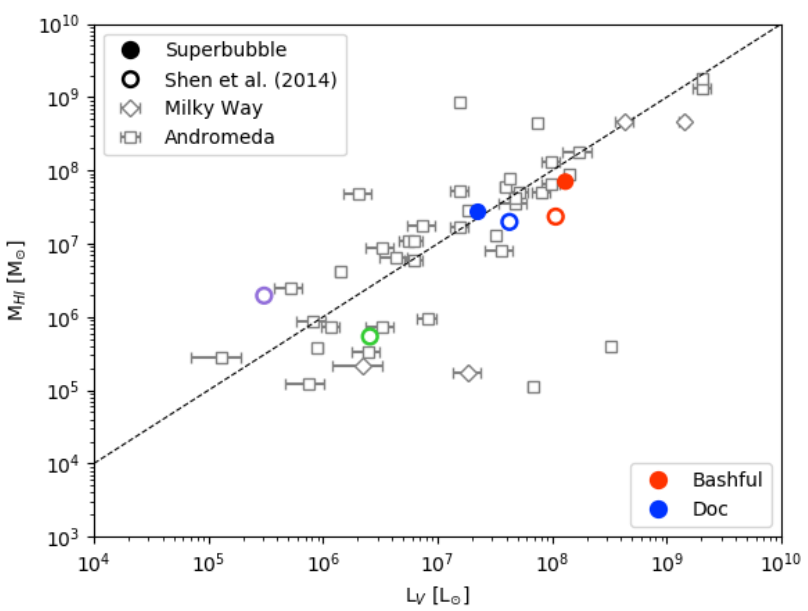

Fig. 6. Total neutral hydrogen (H I) mass versus $V$-band luminosity for the simulated dwarf galaxies. We compute the H I mass directly from the simulation, where the abundance of $\mathrm{HI}$ is calculated by integrating the ionisation equations for primordial species. The filled coloured circles represent Bashful (red) and Doc (blue) in our simulation, at $z=0$. The empty circles with coloured edges correspond to Bashful (red), Doc (blue), Dopey (purple) and Grumpy (green) simulated in Shen et al. (2014) with the blastwave feedback model. The empty symbols represent satellite galaxies of the Milky Way (diamonds) and Andromeda (squares) from the original sample of Local Group galaxies published by McConnachie (2012). The dashed line indicates $L_{V} / M_{\mathrm{HI}}=1$.

$4.9 \mathrm{~km} \mathrm{~s}^{-1}$, respectively, in close agreement with observed $\sigma_{\mathrm{HI}}$ for dIrrs in the Local Group (Stilp et al. 2013). The $v_{\text {rot }} / \sigma$ ratio for the HI disc is 1.78 for Bashful and 3.52 for Doc, significantly higher than the ratios for the stellar discs. Interestingly, the $v_{\text {rot }} / \sigma$ ratio for cold gas is larger in our simulation than in Shen et al. (2014) with the delayed cooling model. The HI gas has higher rotational velocity and lower velocity dispersion, suggesting that the cycle of baryons modulated by the superbubble feedback retains more high angular momentum gas, and generates fewer perturbations in the ISM. We defer a more detailed study of the angular momentum of the accreting gas, outflows, the CGM and the ISM to a future study.

We note that Dopey and Grumpy also have a small amount of neutral hydrogen, of the order of a few times $10^{3} M_{\odot}$, which is significantly less than the total gas mass within the halo (about $\left.10^{7} M_{\odot}\right)$, indicating that most gas is in ionised form. Unlike in Shen et al. (2014), gas in these two halos is generally unable to cool and fuel star formation because of the influence of the neighbouring more massive halos. We discuss this further in Sect. 5 .

\subsection{Mass-metallicity relationship}

In the left panel of Fig. 7, we plot the gas-phase oxygen abundance versus stellar mass for the two simulated dwarfs. In the same figure, we also show the mass-metallicity relation observed over several orders of magnitude in stellar mass, from the brightest spirals to dIrrs (Lee et al. 2006; Woo et al. 2008; Mannucci et al. 2011; Berg et al. 2012; Skillman et al. 2013). We note that the observed mass-metallicity relation holds only for star forming galaxies, as gas-phase oxygen abundances are inferred from emission lines due to photoionization processes from young stars. Also the global shape of the curve strongly depends on the empirical calibration used and, as a consequence, the gas-phase oxygen abundances can differ by up to $0.7 \mathrm{dex}$, 

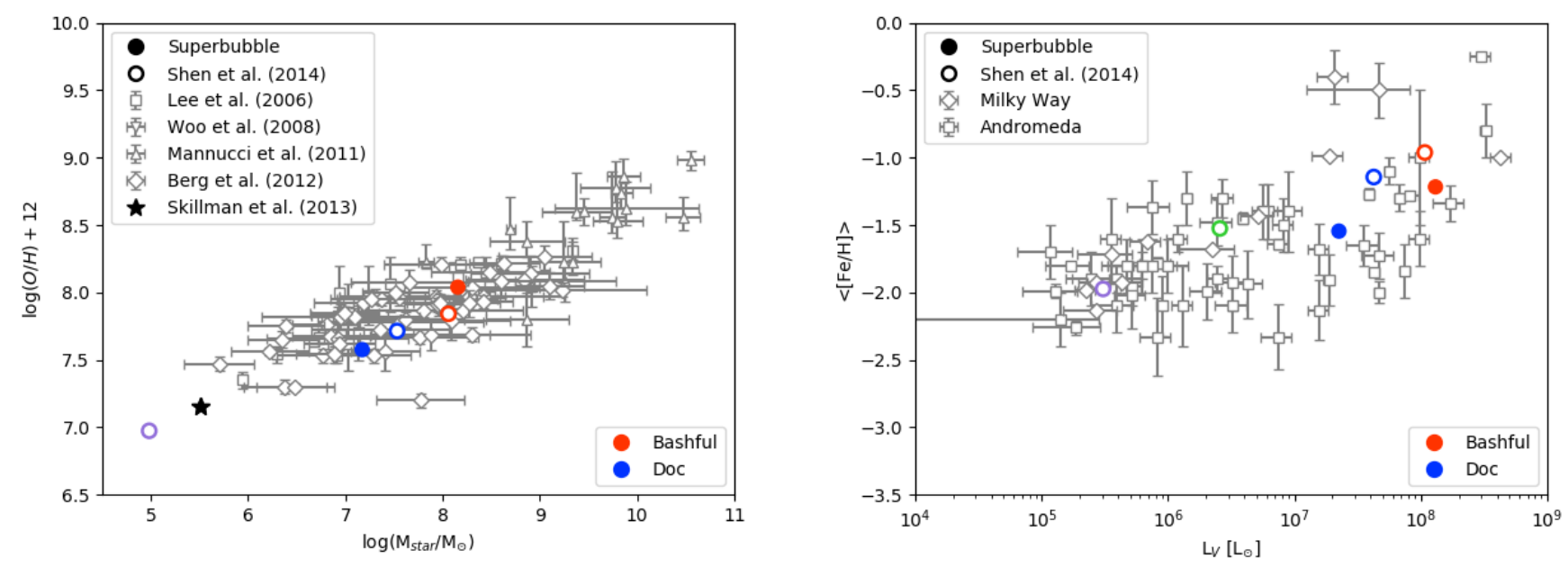

Fig. 7. Left panel: stellar mass-gas phase metallicity relation for low-mass galaxies. The filled coloured circles represent Bashful (red) and Doc (blue) in our simulation, at $z=0$. The empty circles with coloured edges correspond to Bashful (red), Doc (blue), Dopey (purple) and Grumpy (green) simulated in Shen et al. (2014), with the blastwave feedback model. Other symbols show the observed stellar mass-gas phase metallicity relation for nearby dwarf irregulars (empty squares Lee et al. 2006), Local Group dwarfs (empty triangles down Woo et al. 2008), host galaxies of $z<1$ long gamma-ray bursts (empty triangles up, Mannucci et al. 2011), low-luminosity galaxies in the local volume (empty diamonds Berg et al. 2012), and Leo P (black filled star Skillman et al. 2013). Right panel: stellar metallicity vs. $V$-band luminosity for the simulated dwarf galaxies at $z=0$. Our simulated dwarf galaxies and those simulated in Shen et al. (2014) are represented with the same colours and symbols as in right panel. The empty symbols represent satellite galaxies of the Milky Way (diamonds) and Andromeda (squares), from the original sample of Local Group galaxies published by McConnachie (2012).

as shown by Kewley \& Ellison (2008). The gas-phase oxygen abundances estimated for Bashful and Doc follow the measured mass-metallicity relation remarkably well.

In the right panel of Fig. 7, the average metallicity of Bashful and Doc are plotted and compared together with the $V$-band luminosity-metallicity relation measured for the $\mathrm{dSph}$ satellites of the Milky Way, by McConnachie (2012), which spans several dex in luminosity. Although the very low luminosity tail of the observed $V$-band-metallicity relation, corresponding to ultra-faint dwarf galaxies, exhibits a lot of scatter, the luminosity range which brackets our two dwarfs is well constrained. The low luminosity of low-mass galaxies is attributed to the old stellar populations, as opposed to higher mass systems where the majority of the stars formed more recently.

\section{Dark matter}

The inner structure of the dark matter halo hosting dwarf galaxies at the present day is different from what was expected from early predictions. In many studies, such as Walker \& Peñarrubia (2011), Adams et al. (2014), Amorisco \& Evans (2011), Agnello \& Evans (2012), and Oh et al. (2015), it has been claimed that observational data favour shallow dark matter density profiles in the innermost region, in form of cores. Despite the fact that halos in dark matter only simulations exhibit density profiles well fitted by a universal NFW profile (Navarro et al. 1996), recent works have shown how repeated and violent bursts of SN explosions can significantly affect the central gravitational potential wells of dwarf galaxies on short timescales, which transfers kinetic energy from gas to dark matter particles and leads to cusp-core transformation (e.g., Read \& Gilmore 2005; Governato et al. 2010, 2012; Peñarrubia et al. 2012; Pontzen \& Governato 2012; Madau et al. 2014; Oñorbe et al. 2015; Fitts et al. 2017).

In Fig. 8, we plot the present-day dark matter density profiles for Bashful and Doc and we compare them with the corresponding profiles in the DM-only control run. Core formation is

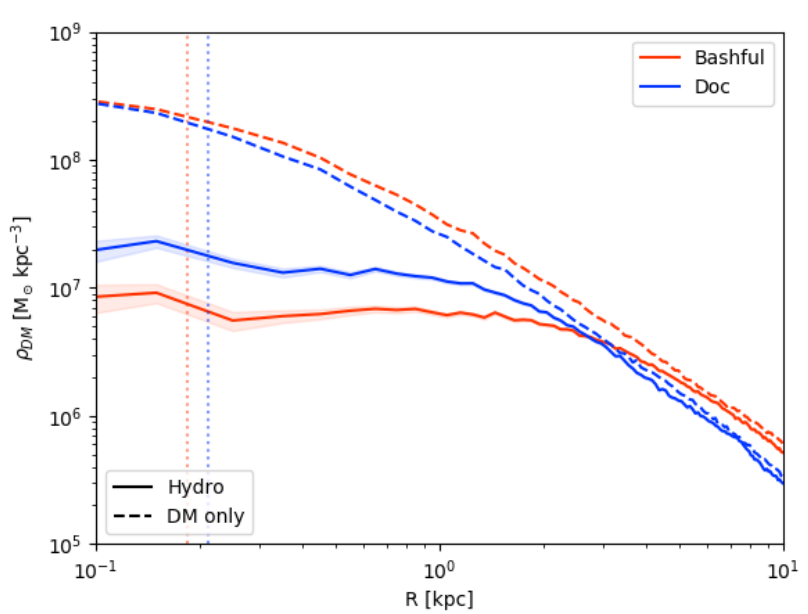

Fig. 8. Present-day dark matter density profiles of Bashful (red) and Doc (blue). The solid lines represent the hydrodynamic simulation, while the dashed lines correspond to the DM only control run. The dotted vertical lines denote the radius within which numerical convergence is not achieved because of two-body relaxation (Power et al. 2003). The shaded bands enclosing the dark matter density profiles correspond to an estimate of the Poisson noise, computed in each radial bin according to the formula $P_{\mathrm{n}}=m_{\mathrm{DM}} * \sqrt{N} / V$, where $N$ is the number of particles in the corresponding bin and $V$ is its volume.

observed in the dark matter profiles for both galaxies even at high redshift. The profiles are well fitted by the pseudo-isothermal fitting formula (Oh et al. 2008):

$\rho_{\mathrm{DM}}(r)=\frac{\rho_{0}}{1+\left(R / R_{c}\right)^{2}}$,

where $\rho_{0}$ and $R_{\mathrm{c}}$ are free parameters and correspond to the central density and the core radius, respectively.

The present day core sizes of Bashful and Doc are $R_{\mathrm{c}}=$ $3.45_{-0.17}^{+0.16} \mathrm{kpc}$ and $R_{\mathrm{c}}=1.86_{-0.07}^{+0.07} \mathrm{kpc}$, respectively. The core size of Bashful is twice as large as the one reported in 

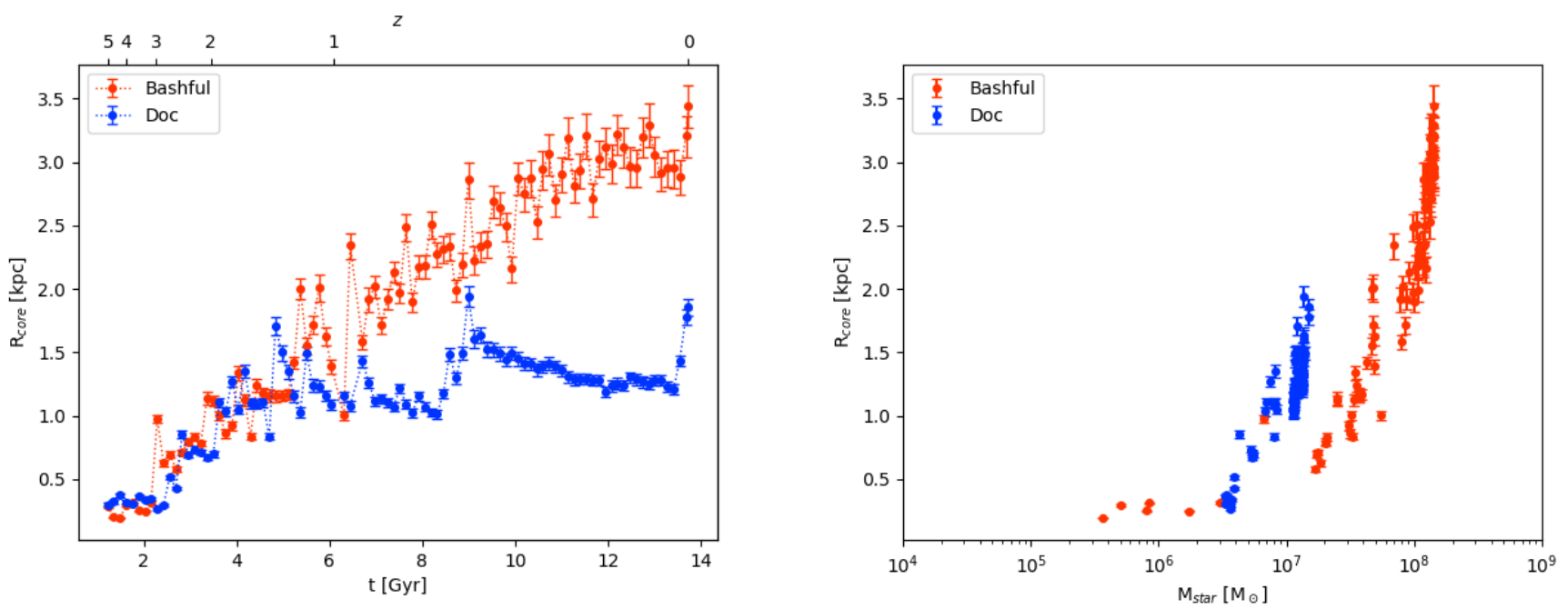

Fig. 9. Left panel: temporal evolution of the core size for the two dwarfs. The rich stellar activity of Bashful (red) leads to a central core which consistently grows in time until it reaches a present-day core radius of $R_{\mathrm{c}}=3.45_{-0.17}^{+0.16} \mathrm{kpc}$. During long quiescence periods, the dark matter core of Doc (blue) remains stable, albeit with a slow decrease in size; its the present day size is $R_{\mathrm{c}}=1.86_{-0.07}^{+0.07} \mathrm{kpc}$. Right panel: evolution of the core radius for Bashful (red) and Doc (blue) as a function of their stellar mass. In order to estimate the error bars for the core radii, we added or subtracted the Poisson noise to or from the dark matter density profile of the two dwarfs and we fitted the resulting profiles for the core radius.

Madau et al. (2014) (1.77 kpc), although it only has about $20 \%$ more stellar mass. Likewise, Doc has a DM core of similar size to that found by Madau et al. (2014) $(2.07 \mathrm{kpc})$ but its total stellar mass is only about half of that found by Madau et al. (2014). The differences in dark matter mass within $3.5 \mathrm{kpc}$ between the dark matter-only run and the hydrodynamic run, $\Delta M_{\mathrm{DM}}$, are $5.79 \times 10^{8} M_{\odot}$ and $2.89 \times 10^{8} M_{\odot}$ for Bashful and Doc, respectively. Furthermore, the 'DM core removal efficiency', defined as $\Delta M_{\mathrm{DM}} / M_{*}$, is 6.55 for Bashful and 19.39 for Doc, which is significantly higher than that found by Madau et al. (2014). This suggests that the superbubble feedback is more effective in creating DM cores.

The evolution of $R_{\mathrm{c}}$ as a function of time (redshift) is shown in the left panel of Fig. 9. Similar to Madau et al. (2014), core formation starts early, because unbinding of the cusp is less energetically demanding when the halo has a shallower potential well. More quantitatively, the minimum energy required for the cusp-core transformation was estimated by Peñarrubia et al. (2012) as $\Delta W / 2 \equiv\left(W_{\text {core }}-W_{\text {cusp }}\right) / 2$, where $W$ is the gravitational binding energy of the dark matter halo,

$W=-4 \pi G \int_{0}^{R_{\text {vir }}} \rho_{\mathrm{DM}} M_{\mathrm{enc}} R \mathrm{~d} R$.

Here, $\rho_{\mathrm{DM}}$ is the dark matter density, while $M_{\text {enc }}$ corresponds to the total mass enclosed in a sphere of radius $R$. We estimated $\Delta W / 2$ and compare this with the total energy injected by Type II SNe $\left(\Delta E_{\mathrm{SN}}\right)$, and find that $\Delta E_{\mathrm{SN}}>\Delta W / 2$ at all times for Bashful and Doc; this again is in agreement with results from Madau et al. (2014). For example, at $z=0,\left(\Delta E_{\mathrm{SN}}, \Delta W / 2\right)=$ $\left(2.6 \times 10^{57} \mathrm{erg}, 4.7 \times 10^{56} \mathrm{erg}\right)$ for Bashful and $\left(2.7 \times 10^{56} \mathrm{erg}, 1.1 \times\right.$ $10^{56} \mathrm{erg}$ ) for Doc. By $z=2$, DM cores of size $\sim 1 \mathrm{kpc}$ have already been in place for both galaxies. At a given redshift, the size of the core and the central density of dark matter halos is strongly correlated with their prior star formation history. In this case, the rich stellar activity of Bashful, with frequent and continuous bursts, allows its core to grow in size until the present day. During long periods of quiescence, the DM core of Doc remains stable, indicating the core-formation process is largely irreversible, as suggested in Pontzen \& Governato (2012). However, the core size does decrease slightly, possibly due to new infalling material with high phase-space density. However, this process takes place over a much longer timescale than the core growth timescale induced by stellar bursts, as shown in the left panel of Fig. 9.

In the right panel of Fig. 9, we plot the evolution of the core radius for Bashful and Doc as a function of their stellar mass. Stellar masses of $M_{*} \gtrsim 10^{6} M_{\odot}$ are enough to produce a central core as large as $\sim 1 \mathrm{kpc}$, which is similar to what is found with the blastwave model Madau et al. (2014), and is in good agreement with the results of Oñorbe et al. (2015) and Fitts et al. (2017). In general, dark matter halos with lower masses also have lower stellar contents, resulting in an insufficient energy input from stellar feedback to relax dark matter density cusps into shallower central densities (Governato et al. 2012; Di Cintio et al. 2014; Madau et al. 2014; Oñorbe et al. 2015). The minimum stellar mass required for the cusp-core transformation and the typical core sizes appear to vary depending on the feedback models employed. The amplitude fluctuations of the central gravitational potential depend on the dynamics of the large-scale galactic outflows, which is determined by the way in which the underlying feedback model operates. The existence of a transition stellar mass below which stellar feedback no longer leads to the formation of dark matter cores is related to the strong dependence between $M_{*}$ and $M_{\mathrm{vir}}$, as halos with only tiny differences in virial mass can vary by orders of magnitude in stellar content. For example, in Sawala et al. (2016) and Revaz \& Jablonka (2018) no dark matter core was found beyond the gravitational softening length of particles, while in Read et al. (2016) dark matter cores were found at all probed halo masses.

To investigate why superbubble feedback is more effective in creating DM cores, we plot the temporal evolution of total baryonic mass within $500 \mathrm{pc}$ of the centre for both runs in Fig. 10. Energetic bursts of star formation shown in Fig. 3 are closely tracked by the cyclic behaviour of the total baryonic mass within $500 \mathrm{pc}$ of the centre of Bashful and Doc. The fluctuations in the central baryonic mass found for our simulated dwarfs have very similar amplitude to the ones found in Madau et al. (2014), but in general the superbubble feedback is more effective in quenching star formation. By explicitly modelling the thermal conduction 


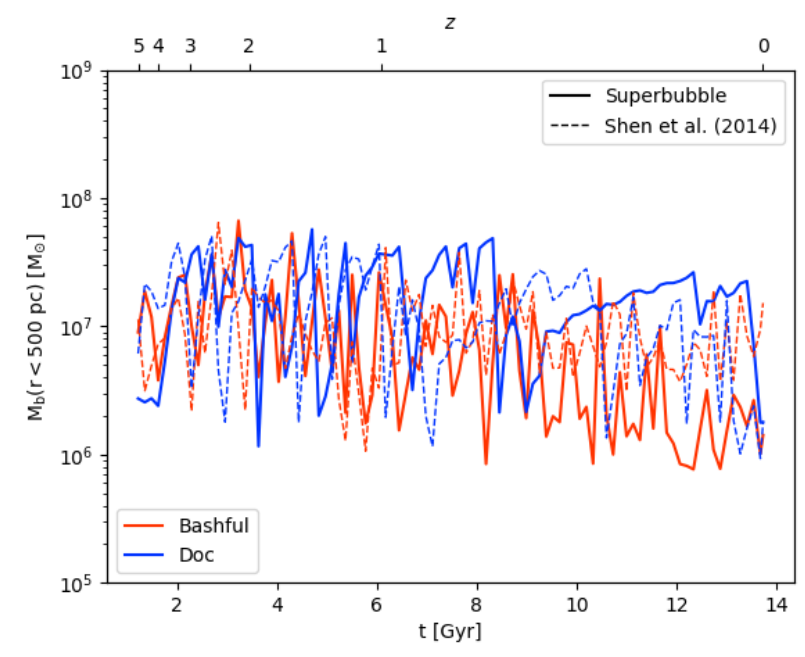

Fig. 10. Temporal evolution of the total baryonic mass enclosed within $500 \mathrm{pc}$ from the centre of the two simulated dwarfs. The solid lines correspond to Bashful (red) and Doc (blue) in our simulation, with the superbubble feedback model. With the same colour convention, the same two dwarfs analysed in Shen et al. (2014) and Madau et al. (2014) are represented with dashed lines.

between the hot and the cold phase of feedback particles, the superbubble model better describes the conversion of thermal energy injected by SNe into kinetic energy. This results in a tighter coupling between the energy released by $\mathrm{SNe}$ and the ISM. This explains why, especially for Doc, in our simulation the total amount of gas mass converted into stars is generally lower than in Madau et al. (2014) at a given redshift, but the fluctuations in the total baryonic content within $500 \mathrm{pc}$ of the centre of the simulated dwarfs is very similar in the two cases. In addition, in our simulation, Bashful has a similar present-day stellar mass to that of the corresponding dwarf in Madau et al. (2014). However, Fig. 3 shows that the stellar activity of Bashful is distributed throughout the entire life of the galaxy and its dark matter core consistently grows until the present day. Instead, in Madau et al. (2014) the stellar activity of the same dwarf is more concentrated within the first $\sim 9 \mathrm{Gyr}$ and, as a consequence, the dark matter core of Bashful no longer grows appreciably. However, it is worth noting that stochasticity in numerical simulations may alter the SFH (e.g., Keller et al. 2019) and hence core formation, growth and final size.

\section{What killed Dopey and Grumpy?}

Dopey and Grumpy, the two faint dwarf galaxies found in Shen et al. (2014), do not form in our simulation. The gas in their halos does not appear to be sufficiently cold or dense to ignite star formation. Although the dark matter halo assembly for Dopey and Grumpy in our simulation is almost identical to that in Shen et al. (2014), the halos accrete less gas, as shown in Fig. 11 (the purple and green lines for Dopey and Grumpy, respectively). The reduction of gas accretion occurs early (around 2-3 Gyr), and lasts throughout cosmic history. As Dopey and Grumpy did not form stars themselves, this reduction is entirely due to feedback from the nearby more massive galaxies, Bashful and Doc. As shown in Keller et al. (2020), the entropy-driven outflows unique to the superbubble feedback generally have higher temperature and higher mass loading at the ISM. Also, because Dopey and Grumpy are only 100-250 kpc away from Bashful and Doc for most of the time (well within the

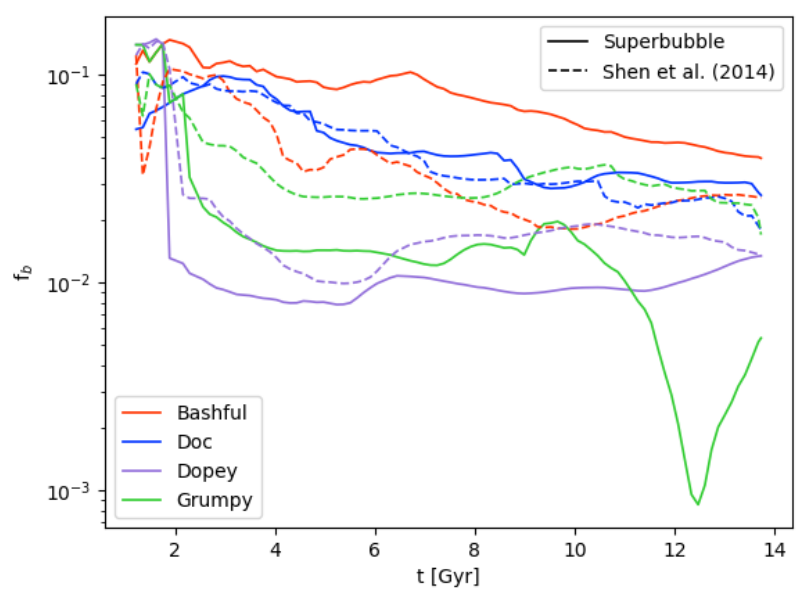

Fig. 11. Temporal evolution of the baryonic fraction $f_{\mathrm{b}} \equiv\left(M_{*}+\right.$ $\left.M_{\text {gas }}\right) / M_{\text {vir }}$ of Bashful (red), Doc (blue), Dopey (purple) and Grumpy (green). The solid lines correspond to the dwarfs simulated with superbubble feedback, while the dashed lines correspond to the dwarfs simulated in Shen et al. (2014) with blastwave feedback.

influence of galactic winds from the two more massive galaxies), the outflows heat the CGM of these small halos to temperatures of the order of the virial value, suppressing their gas accretion, and consequently lowering their halo gas densities, producing longer cooling times (as $t_{\text {cool }} \propto 1 / \rho$ ). In other words, galactic winds from Bashful and Doc serve as a form of 'preventive feedback' for Dopey and Grumpy.

In addition, Dopey and Grumpy are influenced by Bashful and Doc in other ways, which further prevent gas from condensing into the centres of their potential wells. In Fig. 12 we compare the gas properties within the dark halos of both Dopey (upper row) and Grumpy (bottom row) prior to their formation time, with stellar feedback is described by the superbubble (left column) ot the blastwave (right column) model.

\subsection{Dopey - absence of CGM metal transfer}

In Shen et al. (2014), Dopey starts forming the first stars around redshift $z \sim 0.211$, and star formation is triggered by an interaction with a nearby dark halo. The gas accreted by its dark matter halo has a metallicity of order $10^{-2}-10^{-1} Z_{\odot}$ (as shown in the upper right panel of Fig. 12). This is drastically different in our simulation. As shown in the upper left panel of Fig. 12, the halo gas of Dopey is largely primordial at $z=0.211$, which further reduces the cooling rate compared to the blastwave simulation, preventing gas from forming stars. As Dopey has not formed any stars yet in either case, the metal enrichment of Dopey's halo gas is from the CGM of Doc and Bashful. This 'intergalactic metal transfer' is also seen in cosmological simulations of more massive systems (Shen et al. 2012; Muratov et al. 2017; Anglés-Alcázar et al. 2017), but as galactic winds from dwarf galaxies usually have higher masses and metal loading factors (e.g., Christensen et al. 2018), the majority of the metals produced in these galaxies are ejected to the CGM, and therefore metal transfer between CGM is more likely to occur in field dwarf systems. The absence of enriched gas in Dopey's halo in our simulation implies that the extent of the metal-enriched CGM is smaller with the superbubble feedback than with the blastwave model. This is again due to the difference in feedback models. We explore the CGM in more detail in Sect. 6. 

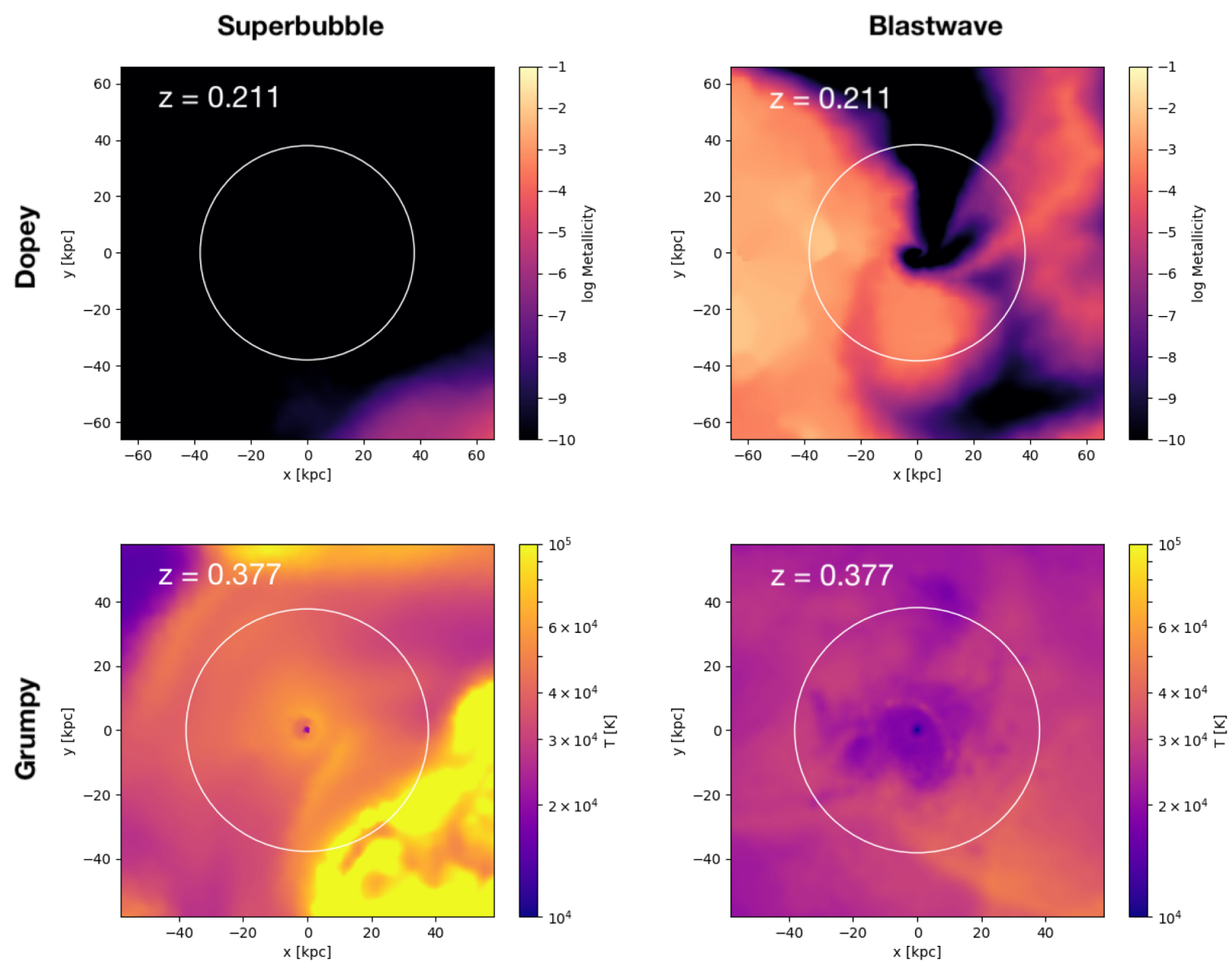

Fig. 12. Upper row: metallicity slice of gas around Dopey's dark halo in our simulation with the superbubble feedback model (left) and in the simulations of Shen et al. (2014), with the blastwave feedback model (right), at redshift $z=0.211$. Bottom row: temperature slice of gas within Grumpy's dark halo in our simulation with the superbubble feedback model (left) and in the simulations of Shen et al. (2014) with the blastwave feedback model (right), at redshift $z=0.377$. The virial radii of Dopey and Grumpy are marked by the white circles.

\subsection{Grumpy - mechanical CGM stripping from nearby dwarf galaxies}

For the case of Grumpy, the situation is somewhat different from that of for Dopey. By the time Grumpy has formed in the previous simulation with the blastwave feedback (at $z \sim 0.377$ ), the halo gas in Grumpy has higher average temperature $(T \sim 4.3 \times$ $\left.10^{4} \mathrm{~K}\right)$ than the blastwave case $\left(T \sim 2.1 \times 10^{4} \mathrm{~K}\right)$ in Shen et al. (2014). As shown in the lower panels of Fig. 12, some gas reaches $\sim 10^{5} \mathrm{~K}$ immediately outside the virial radius. The average halo gas density is also significantly lower, with number density $n \sim 2.4 \times 10^{-5}$ atoms cm$~_{-3}$ instead of $9.4 \times 10^{-5}$ atoms $\mathrm{cm}^{-3}$ in the blastwave run.

In addition to the prevention of gas accretion discussed before, here the heating and dispersion of Grumpy's halo gas are a direct result of the active star formation in Bashful at low redshift in our simulation. Multiple galactic winds driven by SN explosions in Bashful have swept through Grumpy's halo right before $z=0.377$; one is clearly visible in Fig. 12 and an earlier wind was found around $z \sim 0.67$, after a strong starburst in Bashful. The combination of heating and decreased density prevents gas from cooling and condensing into the halo centre and forming stars. As the gas remains diffuse, strong ram-pressure stripping occurs when Grumpy interacts with Doc (as shown in the baryon fraction 'dip' around $12.5 \mathrm{Gyr}$ in Fig. 11). At later times, gas re-accretes, and at $z=0$ the halo has a total gas mass of $M_{\text {gas }}=9.3 \times 10^{6} M_{\odot}$ and a baryon fraction of 0.005 , not very different from Dopey. However the gas remains diffuse and warm, unable to ignite star formation.

In Shen et al. (2014), Dopey and Grumpy lack an old stellar population, and have blue colours and very compact morphologies similar to the properties of extremely metal-deficient compact dwarfs (XBCDs) (Papaderos et al. 2008). The failure of the formation of Dopey and Grumpy in our simulation underlines the fact that the formation of such a low-mass dwarf galaxies is highly stochastic, and likely to be sensitive to physical processes such as gravitational interactions, feedback, ionising radiation from UV background and local sources, and star formation models (e.g., Munshi et al. 2019). However, it is important to mention that our simulation describes a particular case and not all galaxies hosted by halos with $M_{\mathrm{vir}}<10^{10} M_{\odot}$ are influenced by neighbouring galaxies as in our group of dwarfs. It is also worth noting that the lack of pre-reionisation star formation can be an artefact from resolution or the way in which reionisation is modelled in our simulation (UV background is turned on instantaneously). Although in Shen et al. (2014), star formation did not occur even without UV background in a twin simulation by the time reionisation is complete $(z \sim 6)$, higher resolution may enable more realistic gas self-shielding, which allows gas to have runaway cooling and condensation. Some recent 

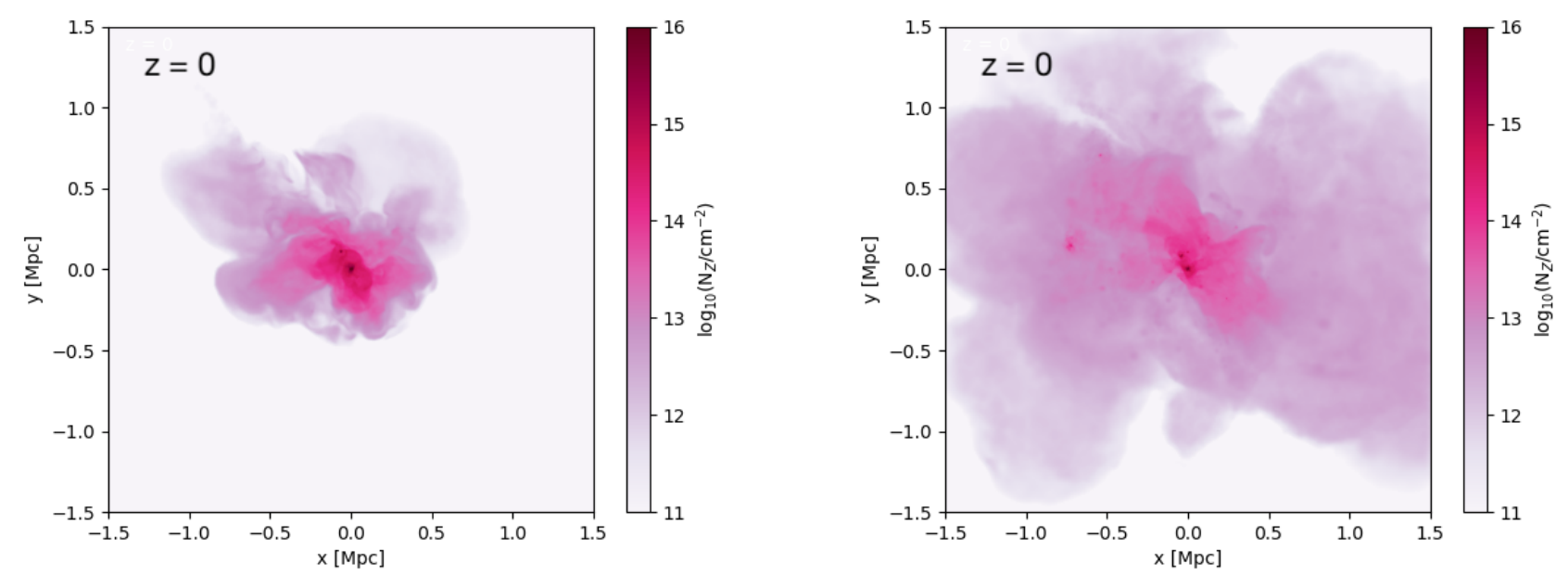

Fig. 13. Present-day column densities for metals computed in a region of $3 \mathrm{Mpc}$ on a side for our simulation with the superbubble feedback model (left panel) and for the one computed in Shen et al. (2014) with the blastwave feedback model (right panel).

works (e.g., Oñorbe et al. 2015; Fitts et al. 2017; Wheeler et al. 2019; Wright et al. 2019b; Applebaum et al., in prep.) indeed form ultra-faint dwarf galaxies with predominately ancient stellar population. We defer detailed investigations of the effects of superbubble feedback, non-uniform UV radiation and selfshielding to a future study, using higher resolution simulations of a larger population of dwarf galaxies.

\section{The circumgalactic medium}

Because of their shallower potential wells, the formation and evolution of dwarf galaxies are highly dependent on how these systems and their surrounding intergalactic medium (IGM) exchange energy, mass and metals. For example, as shown in Sect. 3.5, strong galactic outflows eject $68.8 \%$ and $63.3 \%$ of the metals ever produced in Bashful and Doc, respectively, into the CGM and the IGM. The bursty star formation history (Fig. 3) and the rapid changes in baryonic mass at the inner part of the galaxies (Fig. 10) show that galactic outflows can temporary evacuate the ISM. The mass loading factor of galactic winds at the virial radii, defined as the mass ejection rate per unit of star formation rate $\left(\dot{M}_{\mathrm{w}} / \dot{M}_{*}\right)$, often reaches values of $>10$, much higher than for more massive galaxies (e.g., Shen et al. 2013; Christensen et al. 2018). In turn, the properties of these outflows determine subsequent gas accretion and star formation, and the next cycle of baryonic flows. In this section, we briefly investigate the chemical imprints of the baryon cycles in the multi-phase CGM, and compare our simulation with recent observations from absorption line studies of near field dwarf galaxies. As we show in the following paragraph, the CGM remains a sensitive probe to feedback mechanisms: the ionisation and chemical properties of the CGM in our simulation differ significantly from those in Shen et al. (2014) with the blastwave feedback model.

Figure 13 shows the total column density $\left(N_{Z}\right)$ of metals at $z=0$ for our simulation (left panel) and the result from Shen et al. (2014) with the blastwave feedback model (right panel). The extent of the metal-enriched CGM, defined as the impact parameter from the centre of Bashful at which the covering fraction of $N_{Z}>10^{12} \mathrm{~cm}^{-2}$ drops below $50 \%$, is about $481 \mathrm{kpc}$, approximately 4.7 times Bashful's virial radius $\left(R_{\mathrm{vir}, \mathrm{B}}\right)$ at $z=0$. Galactic outflows are clearly very effective in enriching the CGM. However, the size of the metal bubble is much smaller than that from the blastwave model (which reaches $16 R_{\mathrm{vir}, \mathrm{B}}$ at $z=0$ ), even though the total mass of metals ejected is similar for both simulations. In many ways, the superbubble feedback is less 'ejective' for the halo gas, and this is also reflected in the higher baryonic fractions and higher $M_{\mathrm{HI}} / M_{*}$ (for Bashful and Doc, as shown in Figs. 6 and 11). This is also consistent with the theoretical model proposed by Keller et al. (2020), where the entropy-driven winds have lower velocities.

In addition to the differences in feedback, the extent of metal bubbles is likely also a result of the star formation histories of the two galaxies, especially that of Bashful. In our simulation, Bashful forms about $50 \%$ of its total stellar mass by $z \gtrsim 1$, whereas the blastwave counterpart forms more than $80 \%$ in the same period. Earlier star formation and outflows are more likely to escape the halo potential well and reach larger distances.

In Fig. 14, we compare the column density maps of ionisation species H I, C IV, O VI, and Si II from our simulation and from the simulation in Shen et al. (2014). We compute the ionisation fraction of each element using CLOUDY (Ferland et al. 1998), assuming the gas is under a uniform UV background at $z=0$ from Haardt $\&$ Madau (2012). As the star formation rates at $z=0$ for both galaxies are of order $10^{-2} M_{\odot} \mathrm{yr}^{-1}$, we do not expect internal radiation to have a significant impact the ionisation state of CGM. The column density is computed using the same analysis tool as described in Shen et al. (2013). In Fig. 15 we compare the $1 \mathrm{D}$ column density distribution of these ions as a function of the impact parameter normalised by the galaxies' virial radii $\left(b / R_{\mathrm{vir}}\right)$ with observed values from recent surveys targeting low-mass dwarf galaxies (Bordoloi et al. 2014; Burchett et al. 2016; Johnson et al. 2017; Zheng et al. 2019).

The column densities for different ions exhibit very different distributions. First, we note that neutral hydrogen $(\mathrm{HI})$ is pervasive. At $b / R_{\mathrm{vir}} \sim 1$, the median column density of $\mathrm{HI}$ around our dwarf galaxies is $\mathrm{N}(\mathrm{HI})=1.5 \times 10^{14} \mathrm{~cm}^{-2}$, and the covering fraction for absorption systems with $\mathrm{N}(\mathrm{HI})>10^{13.5} \mathrm{~cm}^{-2}$ within $R_{\mathrm{vir}}$ is $100 \%$. This is consistent with observations, which often find that H I Ly $\alpha$ absorption is ubiquitous around galaxies with a large range of stellar mass (e.g., Prochaska et al. 2011; Werk et al. 2014; Burchett et al. 2016; Bordoloi et al. 2018). For example, Burchett et al. (2016) find a very high detection rate (16 out of 21 ) of systems with $\mathrm{N}(\mathrm{HI})>10^{13.5} \mathrm{~cm}^{-2}$ from the COS-Dwarfs survey for galaxies of stellar mass range $\log M_{*} / M_{\odot}=8-9$. The $1 \mathrm{D}$ column density distribution for $\mathrm{HI}$ from our simulation also shows excellent agreement with the COS-Dwarfs data from Bordoloi et al. (2014) and 
HI CIV OVI

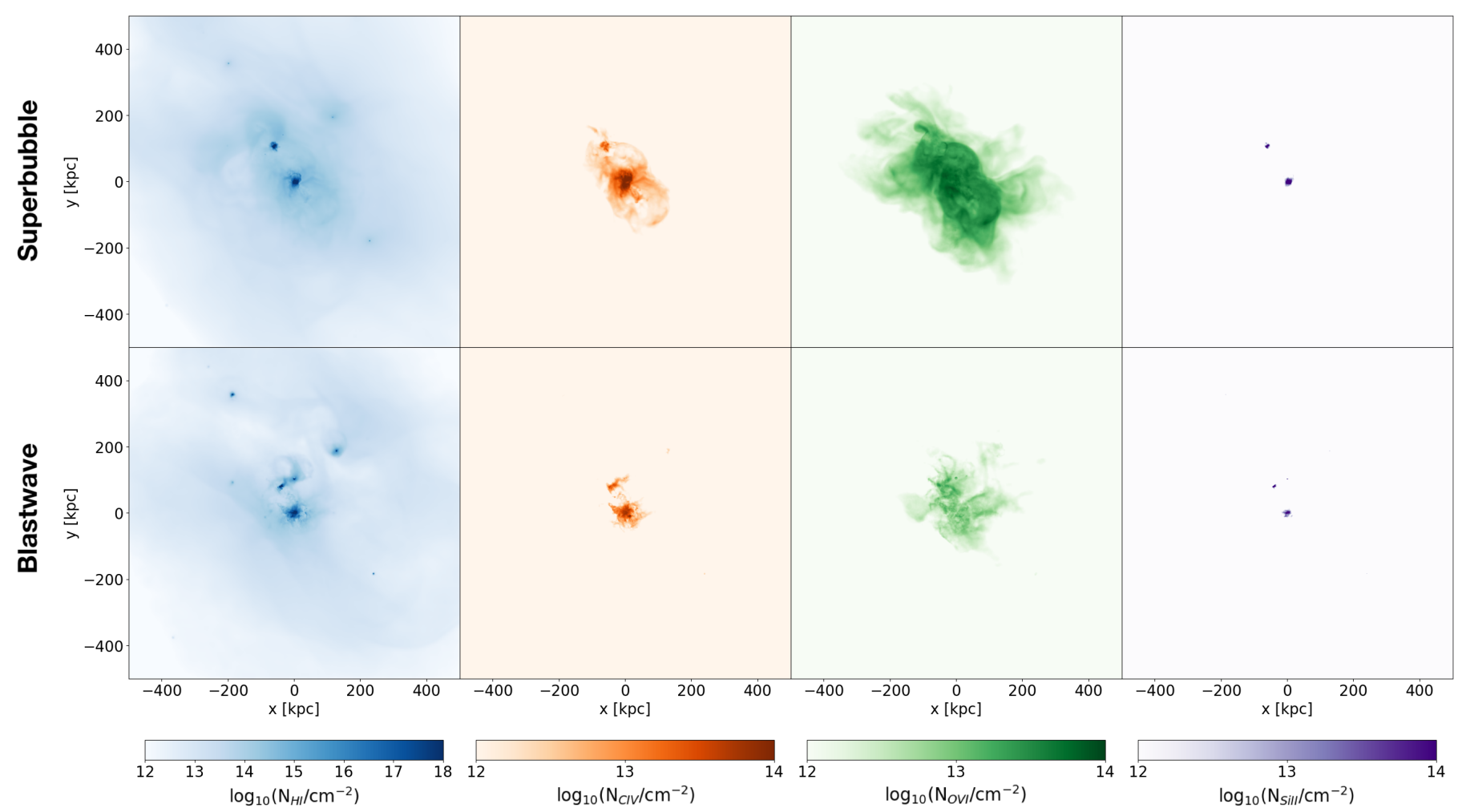

Fig. 14. Projections of the present-day column densities computed in a region of $1 \mathrm{Mpc}$ on a side for ions of different species: $\mathrm{HI}$, C IV, O VI and Si II. Top row: present-day column densities computed for our simulation with the superbubble feedback model and bottom row: ones computed in Shen et al. (2014) with the blastwave feedback model.

Burchett et al. (2016), and LCO/WFCCD data for dwarf galaxies $\left(L<0.1 L_{*}\right)$ from Prochaska et al. (2011). It is interesting to note that the $\mathrm{N}(\mathrm{HI})-b$ relationship seen in the Shen et al. (2014) simulation using the blastwave feedback model is very similar to that found in the present work, which indicates that, unlike metal ions, the distribution of H I around dwarf galaxies is less sensitive to feedback mechanisms (provided that the feedback launches sufficient outflows). The blastwave model does produce a lower H I column density in the CGM, which is consistent with the picture that gas (and metals) is spread to grater distances, as indicated by the metal bubble comparison from Fig. 13. Our results are also consistent with the N(H I) $-b$ relationship for dwarf galaxies from the NIHAO simulation suite Gutcke et al. (2017). The distribution of strong H I absorbers (with $\mathrm{N}(\mathrm{HI}) \gtrsim 10^{15} \mathrm{~cm}^{-2}$ ) appears to be less extended compared to more massive, star-forming galaxies in previous numerical works (e.g., Gutcke et al. 2017; Röttgers et al. 2020). In addition, several recent studies have found that the spacial distribution and covering fractions of the strong $\mathrm{HI}$ absorbing systems can be sensitive to instability processes occurring at smaller scales, and thus sensitive to the CGM-resolution cosmological simulations (e.g., Hummels et al. 2019; Suresh et al. 2019; van de Voort et al. 2019). It is not clear whether the same resolution effect applies to HI absorbers around dwarf galaxies. We defer a more detailed study of the origins of cold H I gas in the CGM and its dependency on resolution to a future paper (Decataldo et al., in prep.).

In contrast to HI, the enriched cold gas appears to be concentrated within the galaxies. For example, the column densities of Si II (tracing cold, $T \sim 10^{4} \mathrm{~K}$ gas) decrease rapidly to below $10^{12} \mathrm{~cm}^{-2}$ within $15.9 \mathrm{kpc}$ from Bashful's centre (i.e. $\left.0.19 R_{\mathrm{vir}, \mathrm{B}}\right)$. This is because the diffuse CGM of low-mass galaxies such as Bashful and Doc is highly ionised by the UV background, and the majority of the metals are in their fully ionised form. The silicon ions tracing the cool diffuse gas, Si II, Si III and Si IV, only account for $27.9 \%$ of the total silicon budget in the CGM. This is also in agreement with recent CGM observations of field dwarf galaxies of similar mass from Johnson et al. (2017), in which the authors found a non-detection of Si II (N(Si II) $\lesssim 10^{12} \mathrm{~cm}^{-2}$ ) around two galaxies (D1 and D2 in their paper) with stellar masses of around $10^{8} M_{\odot}$. The low and intermediate silicon ions ( $\mathrm{Si} 2-4)$ add up to $2 \%-6 \%$ of the total silicon mass. Zheng et al. (2019), on the other hand, did detect Si II at a large impact parameter $(45.2 \mathrm{kpc})$ around WLM (which has $\left.M_{*}=4.3 \times 10^{7} M_{\odot}\right)$, with a column density of around $10^{13} \mathrm{~cm}^{-2}$. A larger sample of observations and simulations of the CGM around faint field dwarf galaxies is probably needed to reach statistically significant conclusions.

The column density distribution of higher ionisation species, such as CIV or O VI, appears to be more extended than Si II. For the moderately ionised CIV, the median column density drops below $10^{13.5} \mathrm{~cm}^{-2}$ (a common detection threshold) at $0.3 R_{\text {vir,B }}$ (about $24.75 \mathrm{kpc}$ ). At greater distances, it appears mostly below the observational points. However, observations from Burchett et al. (2016) show that C IV detection is associated with galaxies of stellar mass $>10^{9.5} M_{\odot}$, and is very rare for lower mass galaxies; this is consistent with what we find here. Our results are also in agreement with the D1 and D2 systems in Johnson et al. (2017), although, again, only below the WLM data point. The column density of highly ionised 

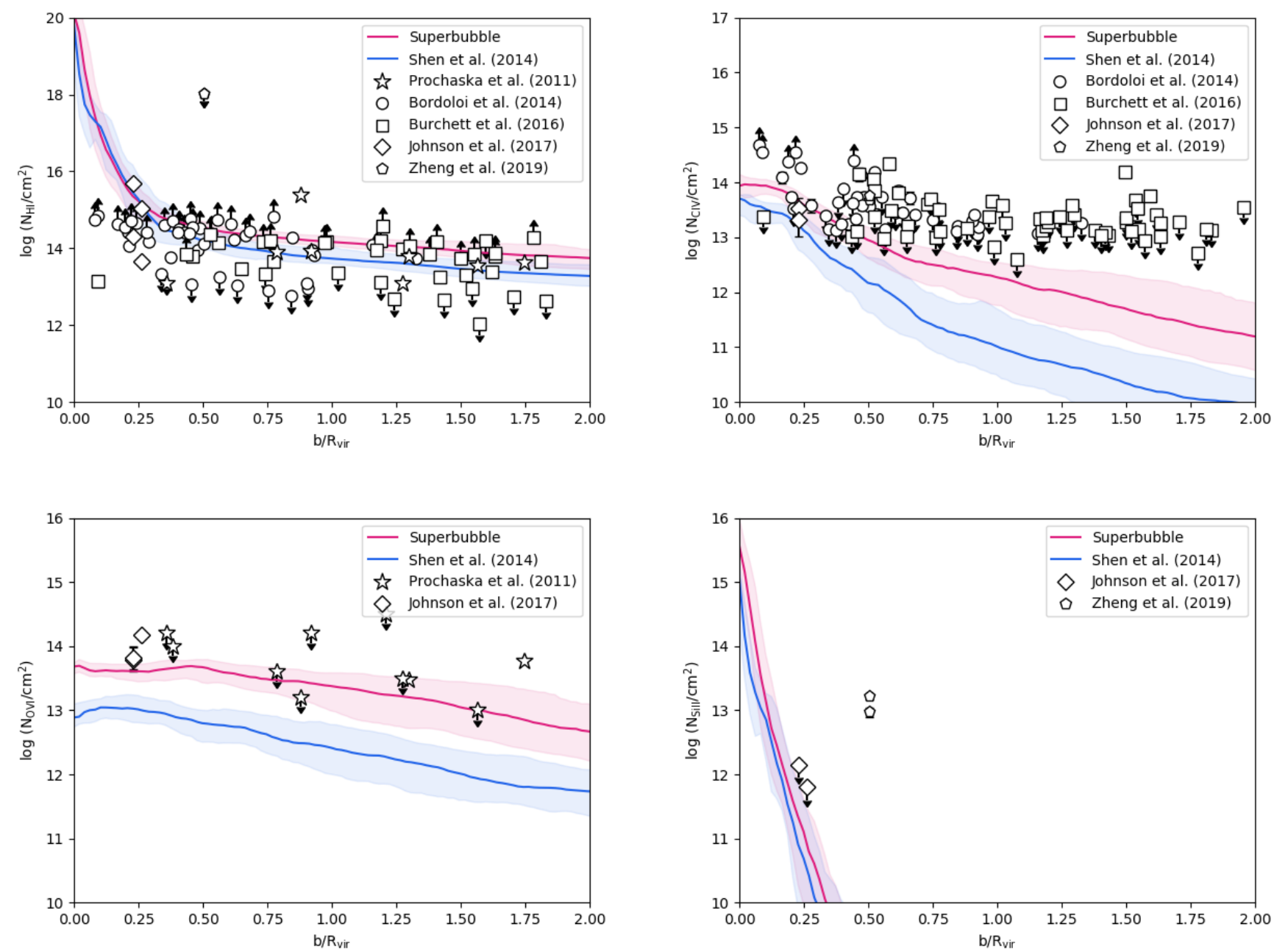

Fig. 15. Present-day column densities for ions of different species: H I (upper left), C IV (upper right), O VI (bottom left) and Si II (bottom right). On the $x$-axis, the impact parameter $b$ is normalised by the virial radius of Bashful. The solid lines represent the present-day column densities computed for our simulation (red) with the superbubble feedback model, and for the simulation presented in Shen et al. (2014) (blue) with the blastwave feedback model. The empty symbols correspond observations of the CGM around a sample of $L<0.1 L_{*}$ dwarf galaxies (empty stars Prochaska et al. 2011), COS-Halos and COS-Dwarfs galaxies (empty circles Bordoloi et al. 2014), nearby faint dwarf galaxies (empty squares Burchett et al. 2016), star-forming field dwarf galaxies (empty diamonds Johnson et al. 2017), and the low-mass dwarf galaxy WLM (empty pentagon Zheng et al. 2019).

O VI decreases more slowly with impact parameter, and remains detectable $\left(\mathrm{N}(\mathrm{O} \mathrm{vI})>10^{13.5} \mathrm{~cm}^{-2}\right)$ up to $\sim R_{\text {vir }}$. Still, the mass in O VI accounts for merely $11.5 \%$ of the total oxygen mass in the CGM. The column densities from our simulation compare reasonably well with those in Johnson et al. (2017), although ours are slightly lower. At larger impact parameter, our results are in close agreement with those of Prochaska et al. (2011) for dwarf galaxies with $L<0.1 L_{*}$. For both C IV and O VI, the column densities from the simulation in Shen et al. (2014) with the blastwave feedback are significantly lower (1 dex or more at larger impact parameters) than those in our simulation. Interestingly, the $\mathrm{O}$ VI distribution from the blastwave run is very similar to that found by Gutcke et al. (2017) for their dwarf galaxy sample, which is not particulatly surprising because the same feedback subgrid model was used. The diffuse CGM temperature is generally around a few times $10^{4} \mathrm{~K}$, as the main channel for producing intermediate and high ions is photoionisation from the UV background. Thus, although the global quantities such as stellar mass, size, kinematics, and total metallicity remain similar for runs with different feedback, the CGM proved to be a more sensitive probe of feedback mechanisms.
In general, although dwarf galaxies eject the majority of their metals into the CGM and the IGM, the column densities of commonly observed metal ions are lower than those associated with more massive galaxies at similar redshift, especially for lower ionisation species (e.g., Hummels et al. 2013; Oppenheimer et al. 2016; Nelson et al. 2018), and this trend is confirmed by observation data. This is likely due to several factors. Firstly, field dwarf galaxies reside in a low-density environment, have lower star formation rates, and produce a smaller amount of metals. Furthermore, these metals are spread over a larger volume, and so the metallicity of the CGM gas is lower. Secondly, both accretion flows and outflows appear to be diffuse. At least in our simulation, there is neither clear filamentary accretion nor clumpy outflows, both of which would increase the column density and covering fraction of low-ionisation lines (e.g., Shen et al. 2013). Thirdly, the low-density environment makes it easier for the gas to be photoionised to higher states, and therefore the common lines only trace a small fraction of the total metal budget. For ions like O VI, the origins around dwarf galaxies may differ significantly from those around $L_{*}$ galaxies, with the latter being dominated by gas heated from feedback or 
accretion shocks cooling through the window of $T \sim 10^{5.5} \mathrm{~K}$ (i.e. peak of O VI abundance in collisional ionisation models) (Roca-Fàbrega et al. 2019; Spilker et al., in prep.). We defer a more quantitative study of the CGM across galaxies with different masses to a forthcoming paper.

\section{Conclusions}

In this work, we present the results of a hydrodynamic 'zoomedin' simulation of dwarf galaxies within the $\Lambda$ CDM model, performed with the code GASOLINE2. The simulation includes a star formation recipe based on the Schmidt law, with a high gas-density threshold, metal-dependent radiative cooling and a uniform UV background, which modifies the ionisation and excitation state of the gas, and a scheme for the turbulent diffusion of metals and thermal energy. The stellar feedback follows the novel superbubble model, which includes a subresolution treatment of multi-phase gas particles, thermal conduction between cold and hot phases, and a model for stochastic evaporation of cold clouds (Keller et al. 2014). The simulation adopts the same initial conditions and numerical setup as in Shen et al. (2014), with the prescription for the SN feedback being the only difference.

Within our simulation volume, only two luminous dwarfs have formed by redshift $z=0$, namely Bashful and Doc. If they were to be observed today, Bashful and Doc would appear blue and be classified as dIrrs. We compared the two simulated dwarfs with the results of Shen et al. (2014) and Madau et al. (2014) in order to qualitatively study the difference between the superbubble and the blastwave feedback models. We also compared them with dwarf galaxies observed in the Local Volume and with the results presented in other recent numerical investigations of galaxy formation and evolution. In particular, we analysed the stellar and cold gas properties of the two luminous dwarf galaxies, their star formation histories, their cusp-core transformation, and the metal enrichment of their surrounding CGM. In addition, we explored why the two faint galaxies formed in Shen et al. (2014) failed to form in less massive halos $\left(M_{\mathrm{vir}}<10^{10} M_{\odot}\right)$ in our simulation.

We summarise the main findings of our work as follows:

- In general, Bashful and Doc have realistic present-day properties. They have low star-formation efficiencies, high coldgas fractions, and low metallicities, closely following the empirical scaling relations found in dwarf galaxies observed in the Local Volume. The superbubble feedback efficiently suppresses star formation in lower mass galaxies, resulting in a factor oftwo less stellar mass in Doc than with the delaycooling model.

- Both Bashful and Doc have highly bursty star formation histories due to the temporary evacuation of the ISM by galactic winds. Although the SFHs are qualitatively similar to the ones of the same dwarfs in Shen et al. (2014), the entropydriven winds from the superbubble feedback are less ejective (at the halo or CGM scale), resulting in a larger baryonic fraction and a larger $M_{\mathrm{HI}} / M_{*}$ ratio for both galaxies. The superbubble feedback also generates less turbulence in the HI disc, leading to a higher $v_{\text {rot }} / \sigma$ ratio.

- The rapid cycles of depletion and re-accretion of gas in the innermost regions of the halos lead to strong fluctuations of the central gravitational potential, causing the central dark matter cusps to flatten into cored density profiles. At $z=0$, the DM core sizes for Bashful and Doc are $3.45_{-0.17}^{+0.16}$ and $1.86_{-0.07}^{+0.07} \mathrm{kpc}$, respectively. The superbubble model appears more effective in destroying dark matter cusps than the previously adopted delayed cooling model. For the same amount of stellar mass formed, the superbubble feedback can remove up to two to three times more mass from the cusp, suggesting a higher coupling efficiency between the energy injected by SNe and the ISM. Likewise, Bashful and Doc also have larger stellar discs in our simulation than in Shen et al. (2014). The strong correlation between the DM core size and the disc scale lengths is consistent with the findings of Peñarrubia et al. (2012) and also with observations (Gentile et al. 2009).

- In Shen et al. (2014), two additional ultra-faint dwarf (UFD) galaxies form, namely Dopey and Grumpy. In our simulation, the two corresponding dark halos never accrete sufficiently cold and dense gas to ignite star formation, and they remain dark due to the influence of Bashful and Doc. The superbubble-driven galactic winds from Bashful and Doc are hotter and more pressurised, which suppresses gas accretion to the nearby halos for Dopey and Grumpy. In addition, cooling time is further increased for Dopey due to an absence of CGM metal transfer (which, in turn, was found in Shen et al. 2014). The halo gas of Grumpy is first swept by winds from Bashful and is then ram-pressure stripped due to the close interactions with Doc, again leading to a longer cooling time and failure of gas condensation. Our results suggest that the formation of UFD galaxies strongly depends on stellar feedback and environmental effects.

- Due to the shallow potential wells of dwarf galaxies, galactic outflows are very effective in enriching the CGM. In our simulation, the metal-enriched CGM extends to about $4.7 R_{\text {vir }}$ of Bashful, significantly smaller than the one in Shen et al. (2014). This again suggests that superbubble feedback is less ejective at the CGM scale (although very effective in ejecting gas at the galaxy centre, as shown in Fig. 10), which is consistent with the analytical model of Keller et al. (2020). The smaller extent of the metal 'bubble' directly results in the aforementioned absence of metals in Dopey's halo.

- The column density distributions of H I, Si II, C IV and O VI as a function of scaled impact parameter $\left(b / R_{\text {vir }}\right)$ are in close agreement with recent observations of CGM around isolated dwarf galaxies. While $\mathrm{H} \mathrm{I}$ is ubiquitous with a covering fraction of unity within the CGM, low and intermediate ions like Si II and C IV are less extended (typically confined within $0.2-0.3 R_{\mathrm{vir}}$ ), and non-detections are common. $\mathrm{O}$ VI is more extended with column density $\mathrm{N}(\mathrm{O}$ VI $) \gtrsim 10^{13.5} \mathrm{~cm}^{-2}$ within $R_{\text {vir }}$, but the mass in O VI is only $11 \%$ of the total CGM oxygen budget, as the diffuse CGM is highly ionised by the UV background. Superbubble feedback produces C IV and O VI column densities that are an order of magnitude higher than those with blastwave feedback.

In summary, the superbubble model better describes the physics behind the $\mathrm{SN}$ feedback process and is capable of forming dwarf galaxies that closely resemble real ones, reproducing the stellar mass and the cold gas content, the bursty star formation histories, the stellar kinematics, and the metallicities of dwarf galaxies observed in the Local Volume. The impact of SN feedback on the host dark matter halos and the metal enrichment of the CGM represent the most sensitive probes of feedback mechanisms.

In future work, we aim to improve the results obtained here, perhaps increasing the simulation volume to have a larger sample of dwarf galaxies.

Acknowledgements. We thank the anonymous referee for the insightful comments and suggestions which helped us to improve the paper. The analysis was partially done with PyNBODY (Pontzen et al. 2013), and we thank Andrew 
Pontzen for providing this very useful analysis tool. We thank Elaad Applebaum, Bernhard Baumschlager, Claudia Cicone, Davide Decataldo and Robert Wissing for very helpful and interesting discussions. S. Shen enjoyed the insightful discussions on the CGM around dwarf galaxies at the virtual KITP Workshop "Fundamentals of Gaseous Halos", and would like to thank the workshop coordinators Cameron Hummels, Benjamin Oppenheimer, Mark Voit and Jessica Werk for providing such an excellent program. The simulations were performed using the resources from the DiRAC HPC facilities in the UK, and the National Infrastructure for HPC and Data Storage in Norway, UNINETT Sigma2 (Project NN9477K). S. Shen acknowledges the support from the Research Council of Norway through NFR Young Research Talents Grant 276043. BW Keller acknowledges funding in the form of a Postodctoral Research Fellowship from the Alexander von Humboldt Siftung. PM acknowledges a NASA contract supporting the WFIRST-EXPO Science Investigation Team (15-WFIRST15-0004), administered by GSFC.

\section{References}

Adams, J. J., Simon, J. D., Fabricius, M. H., et al. 2014, ApJ, 789, 63 Agertz, O., Teyssier, R., \& Moore, B. 2011, MNRAS, 410, 1391 Agertz, O., Pontzen, A., Read, J. I., et al. 2020, MNRAS, 491, 1656 Agnello, A., \& Evans, N. W. 2012, ApJ, 754, L39

Amorisco, N. C., \& Evans, N. W. 2011, MNRAS, 419, 184

Anglés-Alcázar, D., Faucher-Giguère, C.-A., Kereš, D., et al. 2017, MNRAS, 470,4698

Asplund, M., Grevesse, N., Sauval, A. J., \& Scott, P. 2009, ARA\&A, 47, 481

Battaglia, G., Helmi, A., Tolstoy, E., et al. 2008, ApJ, 681, L13

Behroozi, P. S., Wechsler, R. H., \& Conroy, C. 2013, ApJ, 770, 57

Berg, D. A., Skillman, E. D., Marble, A. R., et al. 2012, ApJ, 754, 98

Bergström, L. 2009, New J. Phys., 11, 105006

Bertone, G., Hooper, D., \& Silk, J. 2005, Phys. Rep., 405, 279

Bordoloi, R., Tumlinson, J., Werk, J. K., et al. 2014, ApJ, 796, 136

Bordoloi, R., Prochaska, J. X., Tumlinson, J., et al. 2018, ApJ, 864, 132

Boylan-Kolchin, M., Bullock, J. S., \& Kaplinghat, M. 2011, MNRAS, 415, L40

Boylan-Kolchin, M., Bullock, J. S., \& Kaplinghat, M. 2012, MNRAS, 422, 1203

Brooks, A. M., \& Zolotov, A. 2014, ApJ, 786, 87

Brook, C. B., Stinson, G. S., Gibson, B. K., et al. 2012, MNRAS, 426, 690

Brook, C. B., Di Cintio, A., Knebe, A., et al. 2014, ApJ, 784, L14

Brown, T. M., Tumlinson, J., Geha, M., et al. 2014, ApJ, 796, 91

Burchett, J. N., Tripp, T. M., Bordoloi, R., et al. 2016, ApJ, 832, 124

Chan, T. K., Kereš, D., Oñorbe, J., et al. 2015, MNRAS, 454, 2981

Christensen, C. R., Davé, R., Brooks, A., Quinn, T., \& Shen, S. 2018, ApJ, 867, 142

Cowie, L. L., \& McKee, C. F. 1977, ApJ, 211, 135

Dalla Vecchia, C., \& Schaye, J. 2008, MNRAS, 387, 1431

Di Cintio, A., Brook, C. B., Macciò, A. V., et al. 2014, MNRAS, 437, 415

Dubinski, J., \& Carlberg, R. G. 1991, ApJ, 378, 496

El-Badry, K., Ostriker, E. C., Kim, C.-G., Quataert, E., \& Weisz, D. R. 2019, MNRAS, 490, 1961

Ferland, G. J., Korista, K. T., Verner, D. A., et al. 1998, PASP, 110, 761

Fitts, A., Boylan-Kolchin, M., Elbert, O. D., et al. 2017, MNRAS, 471, 3547

Flores, R. A., \& Primack, J. R. 1994, ApJ, 427, L1

Garrison-Kimmel, S., Boylan-Kolchin, M., Bullock, J. S., \& Lee, K. 2014, MNRAS, 438, 2578

Geha, M., Blanton, M. R., Yan, R., \& Tinker, J. L. 2012, ApJ, 757, 85

Gentile, G., Famaey, B., Zhao, H., \& Salucci, P. 2009, Nature, 461, 627

Girardi, L., Williams, B. F., Gilbert, K. M., et al. 2010, ApJ, 724, 1030

Governato, F., Brook, C., Mayer, L., et al. 2010, Nature, 463, 203

Governato, F., Zolotov, A., Pontzen, A., et al. 2012, MNRAS, 422, 1231

Governato, F., Weisz, D., Pontzen, A., et al. 2015, MNRAS, 448, 792

Griffen, B. F., Ji, A. P., Dooley, G. A., et al. 2016, ApJ, 818, 10

Gutcke, T. A., Stinson, G. S., Macciò, A. V., Wang, L., \& Dutton, A. A. 2017, MNRAS, 464, 2796

Haardt, F., \& Madau, P. 2012, ApJ, 746, 125

Hoeft, M., Yepes, G., Gottlöber, S., \& Springel, V. 2006, MNRAS, 371, 401

Hopkins, P. F., Kereš, D., Oñorbe, J., et al. 2014, MNRAS, 445, 581

Huang, S., Haynes, M. P., Giovanelli, R., et al. 2012, AJ, 143, 133

Hummels, C. B., Bryan, G. L., Smith, B. D., \& Turk, M. J. 2013, MNRAS, 430, 1548

Hummels, C. B., Smith, B. D., Hopkins, P. F., et al. 2019, ApJ, 882, 156

Jethwa, P., Erkal, D., \& Belokurov, V. 2018, MNRAS, 473, 2060

Johnson, S. D., Chen, H.-W., Mulchaey, J. S., Schaye, J., \& Straka, L. A. 2017, ApJ, 850, L10

Kewley, L. J., \& Ellison, S. L. 2008, ApJ, 681, 1183

Keller, B. W., Wadsley, J., Benincasa, S. M., \& Couchman, H. M. P. 2014, MNRAS, 442, 3013

Keller, B. W., Wadsley, J., \& Couchman, H. M. P. 2015, MNRAS, 453, 3499
Keller, B. W., Wadsley, J., \& Couchman, H. M. P. 2016, MNRAS, 463, 1431 Keller, B. W., Wadsley, J. W., Wang, L., \& Kruijssen, J. M. D. 2019, MNRAS, 482, 2244

Keller, B. W., Kruijssen, J. M. D., \& Wadsley, J. W. 2020, MNRAS, 493, 2149 Kimm, T., \& Cen, R. 2014, ApJ, 788, 121

Kirby, E. N., Cohen, J. G., Guhathakurta, P., et al. 2013, ApJ, 779, 102

Kirby, E. N., Bullock, J. S., Boylan-Kolchin, M., Kaplinghat, M., \& Cohen, J. G. 2014, MNRAS, 439, 1015

Kroupa, P. 2001, MNRAS, 322, 231

Kuhlen, M., Madau, P., \& Silk, J. 2009, Science, 325, 970

Leaman, R., Venn, K. A., Brooks, A. M., et al. 2012, ApJ, 750, 33

Lee, H., Skillman, E. D., Cannon, J. M., et al. 2006, ApJ, 647, 970

Łokas, E. L., Kazantzidis, S., \& Mayer, L. 2011, ApJ, 739, 46

Mac Low, M.-M., \& McCray, R. 1988, ApJ, 324, 776

Madau, P., Kuhlen, M., Diemand, J., et al. 2008, ApJ, 689, L41

Madau, P., Shen, S., \& Governato, F. 2014, ApJ, 789, L17

Mannucci, F., Salvaterra, R., \& Campisi, M. A. 2011, MNRAS, 414, 1263

Marigo, P., Girardi, L., Bressan, A., et al. 2008, A\&A, 482, 883

Mashchenko, S., Wadsley, J., \& Couchman, H. M. P. 2008, Science, 319, 174

McConnachie, A. W. 2012, AJ, 144, 4

Moore, B. 1994, Nature, 370, 629

Moore, B., Ghigna, S., Governato, F., et al. 1999, ApJ, 524, L19

Moster, B. P., Naab, T., \& White, S. D. M. 2013, MNRAS, 428, 3121

Munshi, F., Governato, F., Brooks, A. M., et al. 2013, ApJ, 766, 56

Munshi, F., Brooks, A. M., Christensen, C., et al. 2019, ApJ, 874, 40

Muratov, A. L., Kereš, D., Faucher-Giguère, C.-A., et al. 2017, MNRAS, 468, 4170

Nath, B. B., \& Shchekinov, Y. 2013, ApJ, 777, L12

Navarro, J. F., Frenk, C. S., \& White, S. D. M. 1996, ApJ, 462, 563

Nelson, D., Kauffmann, G., Pillepich, A., et al. 2018, MNRAS, 477, 450

Noh, Y., \& McQuinn, M. 2014, MNRAS, 444, 503

Oh, S.-H., de Blok, W. J. G., Walter, F., Brinks, E., \& Kennicutt, R. C. 2008, AJ, 136,2761

Oh, S.-H., Hunter, D. A., Brinks, E., et al. 2015, AJ, 149, 180

Okamoto, T., Gao, L., \& Theuns, T. 2008, MNRAS, 390, 920

Oppenheimer, B. D., \& Davé, R. 2006, MNRAS, 373, 1265

Oppenheimer, B. D., Crain, R. A., Schaye, J., et al. 2016, MNRAS, 460, 2157

Oñorbe, J., Boylan-Kolchin, M., Bullock, J. S., et al. 2015, MNRAS, 454, 2092

Papaderos, P., Guseva, N. G., Izotov, Y. I., \& Fricke, K. J. 2008, A\&A, 491, 113

Peñarrubia, J., Pontzen, A., Walker, M. G., \& Koposov, S. E. 2012, ApJ, 759, L42

Pontzen, A., \& Governato, F. 2012, MNRAS, 421, 3464

Pontzen, A., Roškar, R., Stinson, G., \& Woods, R. 2013, Astrophysics Source Code Library [record ascl:1305.002]

Power, C., Navarro, J. F., Jenkins, A., et al. 2003, MNRAS, 338, 14

Prochaska, J. X., Weiner, B., Chen, H. W., Mulchaey, J., \& Cooksey, K. 2011, ApJ, 740, 91

Raiteri, C. M., Villata, M., \& Navarro, J. F. 1996, A\&A, 315, 105

Read, J. I., \& Gilmore, G. 2005, MNRAS, 356, 107

Read, J. I., Agertz, O., \& Collins, M. L. M. 2016, MNRAS, 459, 2573

Read, J. I., Iorio, G., Agertz, O., \& Fraternali, F. 2017, MNRAS, 467, 2019

Revaz, Y., \& Jablonka, P. 2018, A\&A, 616, A96

Roca-Fàbrega, S., Dekel, A., Faerman, Y., et al. 2019, MNRAS, 484, 3625

Röttgers, B., Naab, T., Cernetic, M., et al. 2020, MNRAS, 496, 152

Sawala, T., Frenk, C. S., Fattahi, A., et al. 2016, MNRAS, 457, 1931

Schmidt, M. 1959, ApJ, 129, 243

Sharma, P., Roy, A., Nath, B. B., \& Shchekinov, Y. 2014, MNRAS, 443, 3463

Shen, S., Wadsley, J., \& Stinson, G. 2010, MNRAS, 407, 1581

Shen, S., Madau, P., Aguirre, A., et al. 2012, ApJ, 760, 50

Shen, S., Madau, P., Guedes, J., et al. 2013, ApJ, 765, 89

Shen, S., Madau, P., Conroy, C., Governato, F., \& Mayer, L. 2014, ApJ, 792, 99 Simpson, C. M., Bryan, G. L., Johnston, K. V., et al. 2013, MNRAS, 432, 1989

Skillman, E. D., Salzer, J. J., Berg, D. A., et al. 2013, AJ, 146, 3

Smagorinsky, J. 1963, Mon. Weather Rev., 91, 99

Smith, M. C., Sijacki, D., \& Shen, S. 2018, MNRAS, 478, 302

Springel, V., \& Hernquist, L. 2003, MNRAS, 339, 289

Springel, V., Wang, J., Vogelsberger, M., et al. 2008, MNRAS, 391, 1685

Stadel, J., Potter, D., Moore, B., et al. 2009, MNRAS, 398, L21

Steffen, F. D. 2009, Eur. Phys. J. C, 59, 557

Steinwandel, U. P., Moster, B. P., Naab, T., Hu, C.-Y., \& Walch, S. 2020, MNRAS, 495, 1035

Stilp, A. M., Dalcanton, J. J., Warren, S. R., et al. 2013, ApJ, 765, 136

Stinson, G., Seth, A., Katz, N., et al. 2006, MNRAS, 373, 1074

Suresh, J., Nelson, D., Genel, S., Rubin, K. H. R., \& Hernquist, L. 2019, MNRAS, 483, 4040

Teyssier, R., Pontzen, A., Dubois, Y., \& Read, J. I. 2013, MNRAS, 429, 3068

Tollet, E., Macciò, A. V., Dutton, A. A., et al. 2016, MNRAS, 456, 3542

Tolstoy, E., Hill, V., \& Tosi, M. 2009, ARA\&A, 47, 371 
M. Mina et al.: The baryon cycle of Seven Dwarfs with superbubble feedback

Trujillo-Gomez, S., Klypin, A., Colín, P., et al. 2015, MNRAS, 446, 1140 van de Voort, F., Springel, V., Mandelker, N., van den Bosch, F. C., \& Pakmor, R. 2019, MNRAS, 482, L85

Wadsley, J. W., Keller, B. W., \& Quinn, T. R. 2017, MNRAS, 471, 2357

Walker, M. G., \& Peñarrubia, J. 2011, ApJ, 742, 20

Weisz, D. R., Dalcanton, J. J., Williams, B. F., et al. 2011, ApJ, 739, 5

Weisz, D. R., Dolphin, A. E., Skillman, E. D., et al. 2014, ApJ, 789, 147

Werk, J. K., Prochaska, J. X., Tumlinson, J., et al. 2014, ApJ, 792, 8

Wetzel, A. R., Hopkins, P. F., Kim, J.-H., et al. 2016, ApJ, 827, L23
Wheeler, C., Oñorbe, J., Bullock, J. S., et al. 2015, MNRAS, 453, 1305 Wheeler, C., Hopkins, P. F., Pace, A. B., et al. 2019, MNRAS, 490, 4447 Woo, J., Courteau, S., \& Dekel, A. 2008, MNRAS, 390, 1453

Wright, A. C., Brooks, A. M., Weisz, D. R., \& Christensen, C. R. 2019a, MNRAS, 482, 1176

Wright, R. J., Lagos, C. D. P., Davies, L. J. M., et al. 2019b, MNRAS, 487, 3740

Zheng, Y., Putman, M. E., Emerick, A., et al. 2019, MNRAS, 490, 467

Zolotov, A., Brooks, A. M., Willman, B., et al. 2012, ApJ, 761, 71 